Article

\title{
Converter-Driven Stability Analysis of Power Systems Integrated with Hybrid Renewable Energy Sources
}

\author{
Jianqiang Luo ${ }^{1} \mathbb{D}$, Yiqing Zou ${ }^{1}$, Siqi Bu ${ }^{1,2,3, * \mathbb{D}}$ and Ulas Karaagac ${ }^{1}$ \\ 1 Department of Electrical Engineering, The Hong Kong Polytechnic University, Kowloon 999077, Hong Kong; \\ jianqiang.luo@polyu.edu.hk (J.L.); 18095429g@connect.polyu.hk (Y.Z.); ulas.karaagac@polyu.edu.hk (U.K.) \\ 2 Shenzhen Research Institute, The Hong Kong Polytechnic University, Shenzhen 518057, China \\ 3 Research Institute for Smart Energy, The Hong Kong Polytechnic University, Kowloon 999077, Hong Kong \\ * Correspondence: siqi.bu@polyu.edu.hk
}

check for updates

Citation: Luo, J.; Zou, Y.; Bu, S.; Karaagac, U. Converter-Driven Stability Analysis of Power Systems Integrated with Hybrid Renewable Energy Sources. Energies 2021, 14, 4290. https://doi.org/10.3390/ en14144290

Academic Editor: Juri Belikov

Received: 3 May 2021

Accepted: 3 June 2021

Published: 16 July 2021

Publisher's Note: MDPI stays neutral with regard to jurisdictional claims in published maps and institutional affiliations.

Copyright: (c) 2021 by the authors. Licensee MDPI, Basel, Switzerland. This article is an open access article distributed under the terms and conditions of the Creative Commons Attribution (CC BY) license (https:// creativecommons.org/licenses/by/ $4.0 /)$.
Abstract: Renewable energy sources such as wind power and photovoltaics (PVs) have been increasingly integrated into the power system through power electronic converters in recent years. However, power electronic converter-driven stability has issues under specific circumstances, for instance, modal resonances might deteriorate the dynamic performance of the power systems or even threaten the overall stability. In this work, the integration impact of a hybrid renewable energy source (HRES) system on modal interaction and converter-driven stability was investigated in an IEEE 16-machine 68-bus power system. In this paper, firstly, an HRES system is introduced, which consists of full converter-based wind power generation (FCWG) and full converter-based photovoltaic generation (FCPV). The equivalent dynamic models of FCWG and FCPV are then established, followed by linearized state-space modeling. On this basis, converter-driven stability analysis was performed to reveal the modal resonance mechanisms between different renewable energy sources (RESs) and weak grids in the interconnected power systems and the multi-modal interaction phenomenon. Additionally, time-domain simulations were conducted to verify the effectiveness of dynamic models and support the converter-driven stability analysis results. To avoid detrimental modal resonances, a multi-modal and multi-parametric optimization strategy is further proposed by retuning the controller parameters of the multi-RESs in the HRES system. The overall results demonstrate the modal interaction effect between the external AC power system and the HRES system and its various impacts on converter-driven stability.

Keywords: converter-driven stability; hybrid renewable energy source (HRES) system; multi-modal interaction; full converter-based wind power generation (FCWG); full converter-based photovoltaic generation (FCPV)

\section{Introduction}

High penetration of converter-based power sources has become a popular trend due to its benefits in terms of environmental protection and social sustainability, especially the integration of wind power and photovoltaic (PV) solar energy in modern power systems [1,2]. The full converter-based wind generation (FCWG, e.g., permanent magnet synchronous generator (PMSG)) is more promising than doubly-fed induction generators (DFIGs) in new wind power applications [3]. As for PV solar energy, several generic PV system models based on the Type 4 wind turbine generator model have been introduced by Western Electric Coordinating Council (WECC) [4]. PV generation is modeled as an inverter-based generator associated with a variety of active power control reactive power control options.

Renewable energy sources are interconnected to the power system via flexibly controlled power electronic converters that might produce new stability issues due to the modal interactions between converter-based generators and the power system, such as converter-driven stability and resonance stability [5]. In particular, oscillation issues could 
be induced by the modal interaction between converters and external AC power systems. The sub-synchronous resonance (SSR) was observed in ERCOT of United States [6], Hebei province of North China [7], London blackout in the United Kingdom [8], and sub-synchronous oscillation (SSO) in Xinjiang province of China [9]. Low-frequency power oscillations are normally caused by the modal interaction between the interconnection of power grids and the fast-response automatic voltage regulators (AVRs). It is generally understood that the occurrence of the oscillations is due to the lack of damping of power systems electromechanical oscillation modes (EOMs) $[10,11]$. An unusual transition in electromechanical dynamics is disclosed in [12], which indicates that an EOM may be dominated by FCWG dynamics and tuned into a quasi-EOM when the FCWG quasielectromechanical state variables actively participate. The authors of [13] studied how system impedance and the parameters of the phase-locked loop (PLL) affect the dynamic behavior and the stability limits of the converters in HVDC applications.

Furthermore, increasingly power electronic converter-interfaced renewable energy sources introduce a challenge for converter-driven stability of the overall system [14]. Although the interconnection of power electronic converter-interfaced renewable generators and conventional power systems enhances the overall flexibility and controllability [15-17], the modal interactions of both transmission and distribution systems become complicated [18-20]. In contrast to the traditional synchronous generators (SGs), converter-based renewable energy sources have a major impact on power system converter-driven stability. The authors of [21] studied how the parameters of rotor current controllers have influences on the eigenvalues shift locus. Based on the dynamic modeling and analysis of traditional generators and converter-based DFIG, the impact on power system stability is the minimum under small-scale penetration of wind power generations [22]. However, when the wind power penetration level increases, the converter-driven stability of the overall power systems may be greatly affected. The authors of [23] conclude that the damping reduction of power system EOM may occur at weak interconnection lines and increased wind power penetration level. In multiple grid-interconnected PV generation systems, the coupling behavior between PLLs and near converters may make the system more vulnerable or even lose converter-driven stability [24,25].

In this work, a hybrid renewable energy source (HRES) system consisting of FCWG and FCPV was integrated into the same power system to study the complex modal interaction with external AC power systems and thus consequent impact on converter-driven stability. The main contributions of this paper are listed as follows:

1. Detailed dynamic models of FCWG and FCPV, including PMSG, PV generation unit, DC/DC converter and the associated control system, DC-link, grid side converter (GSC) and associated control systems, synchronous reference frame phase-locked loop (SRF-PLL), and the external AC power system are established. The linearized state-space models of each dynamic component, as well as the entire closed-loop system, are developed as the foundation of converter-driven stability analysis.

2. Based on the above models, modal analysis is conducted with different wind power and PV solar energy penetration levels in the IEEE 16-machine 68-bus system. Peculiarly, open-loop FCWG oscillation mode (FOM) and FCPV oscillation mode (POM) are tuned to be close to critical EOM in terms of frequency, which is the necessary condition of open-loop modal resonance.

3. Open-loop and closed-loop modal analysis is compared. Multi-modal interaction in the examined system with different renewable energy penetration levels is evaluated to analyze the essential resonance mechanism, which provides a theoretical indication to alleviate the negative effect caused by strong modal resonance.

4. To circumvent the malignant modal resonance and to enhance the converter-driven stability, a modal interaction optimization strategy was implemented to prevent potential modal resonance through carefully retuning the controller parameters of the HRES system. The overall converter-driven stability and dynamic performance of the entire system were improved thereafter. 


\section{Hybrid Renewable Energy Source (HRES) System}

Wind power and PV solar energy have become prevalent renewable power sources and represent a gradually increasing share of generation in modern power systems. Consequently, these renewable energy sources also induce a long-lasting and complex impact on power system stability [26]. In this section, an HRES system is introduced to cover different types of renewable energy and their complex modal interaction with external AC power systems.

\subsection{Configuration of FCWG}

The typical topology of an FCWG is depicted in Figure 1.

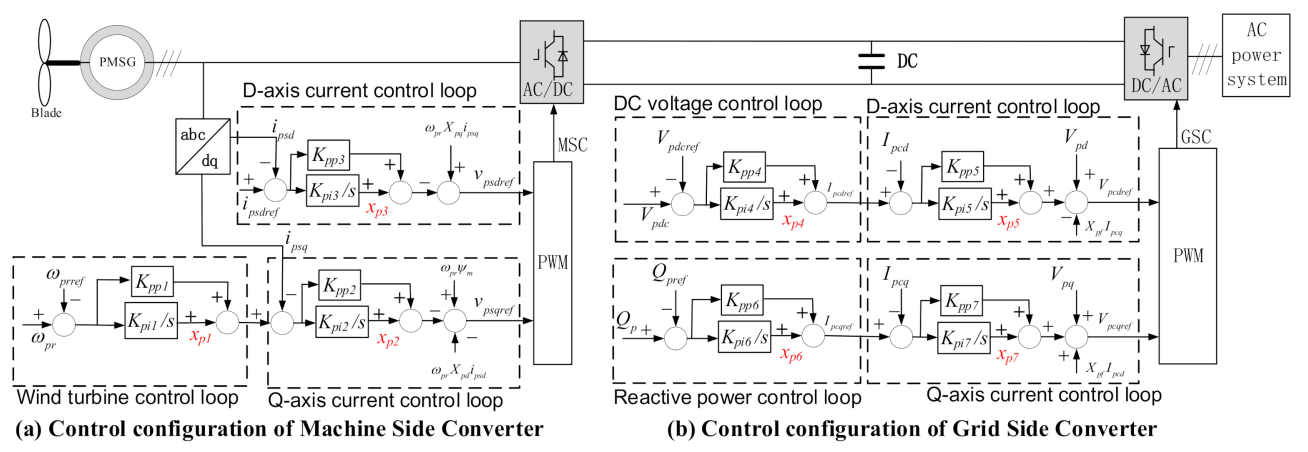

Figure 1. Physical configuration of an FCWG connected to the AC power system.

The FCWG consists of three parts: (1) the PMSG, the machine side converter (MSC), and the associated control system (as demonstrated in Figure 1a); (2) the DC-link, the grid side converter (GSC), and the associated control system (as shown in Figure 1b); and (3) the SRF-PLL (as presented in Figure 2), which is used to synchronize FCWG with the external power system.

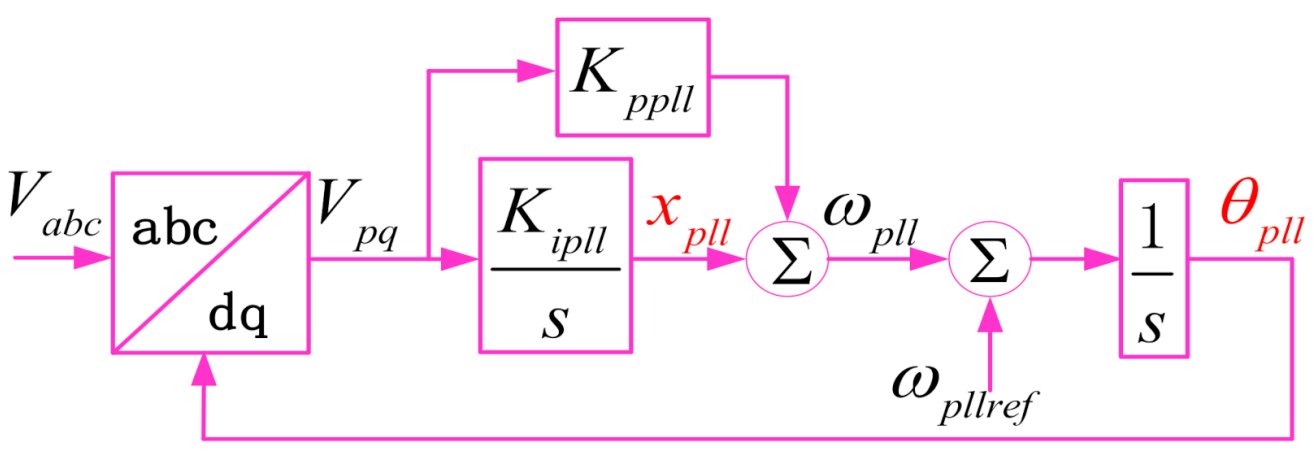

Figure 2. Block diagram of SRF-PLL.

\subsection{Configuration of FCPV}

A full converter-based photovoltaic generation (FCPV) farm interconnected to a power system is depicted in Figure 3, which consists of three main parts: (1) a PV generation unit, the DC/DC converter, and the associated control system; (2) the DC-link, GSC, and the associated control system; and (3) the SRF-PLL, which has the same control configuration and function as that of FCWG. 


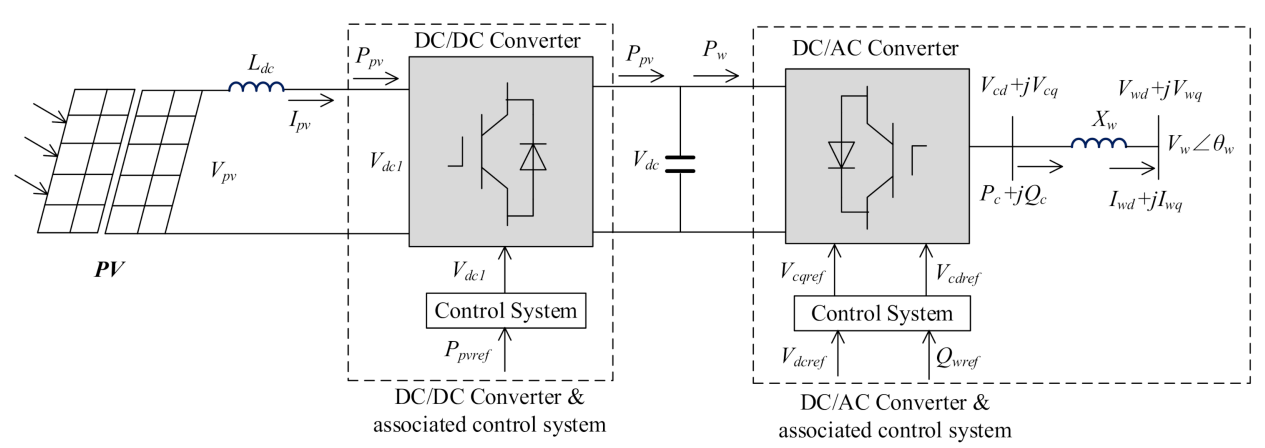

Figure 3. Physical configuration of an FCPV interconnected to the AC power system.

\section{Dynamic Models of FCWG and FCPV}

To elaborate the modal interaction mechanism, the external AC power system excluding the HRES dynamics is denoted as the open-loop system, while the entire system is the closed-loop system. Therefore, the impact of the HRES system can be quantified through open-loop and closed-loop modal analysis.

\subsection{State-Space Model of FCWG}

Dynamics equations of all FCWG components are derived in this section.

1. Modeling of PMSG

The PMSG can be represented by the following equations:

$$
\begin{aligned}
& \left\{\begin{array}{l}
\frac{d \psi_{p s d}}{d t}=-\omega_{0} R_{p s} i_{p s d}-\omega_{0} v_{p s d}+\omega_{0} \omega_{p r} \psi_{p s q} \\
\frac{d \psi_{p s q}}{d t}=-\omega_{0} R_{p s} i_{p s q}-\omega_{0} v_{p s q}-\omega_{0} \omega_{p r} \psi_{p s q}
\end{array}\right. \\
& \left\{\begin{array}{l}
\psi_{p s d}=X_{p d} i_{p s d}-\psi_{p m} \\
\psi_{p s q}=X_{p q} i_{p s q}
\end{array}\right.
\end{aligned}
$$

where $\psi_{p s k}, v_{p s k}, i_{p s k}$ and $X_{p k}(\mathrm{k}=\mathrm{d}, \mathrm{q})$ are the direct and quadrature axis flux, voltage, current, and reactance of stator winding, respectively. $R_{p s}$ is the stator winding resistance, $\omega_{b}$ is the base angular speed in rad/s, $\psi_{p m}$ is the flux linkage produced by the permanent magnet. $\omega_{p r}$ is the stator electrical angular speed, defined by: $\omega_{p r}=n_{p} \omega_{m}=\omega_{m}$, where $n_{p}=1$ is the number of pole pairs of PMSG and $\omega_{m}$ is the mechanical (rotor) angular speed.

The motion equation of the wind turbine rotor is expressed as

$$
H_{p r} \frac{d \omega_{p r}}{d t}=T_{p m}-T_{p e}
$$

where $H_{p r}$ is the inertia constant of the rotor, $T_{p m}$ is the mechanical torque of the wind turbine, $T_{p e}$ is the electrical torque of PMSG.

2. Modeling of MSC

From Figure 1a, the dynamics of MSC are derived as

$$
\left\{\begin{array}{l}
i_{p s q r e f}=K_{p p 1}\left(\omega_{p r}-\omega_{p r r e f}\right)+x_{p 1} \\
\frac{d x_{p 1}}{d t}=K_{p i 1}\left(\omega_{p r}-\omega_{p r r e f}\right) \\
\frac{d x_{p 2}}{d t}=K_{p i 2}\left(i_{p s q r e f}-i_{p s q}\right) \\
\frac{d x_{p 3}}{d t}=K_{p i 3}\left(i_{p s d r e f}-i_{p s d}\right)
\end{array}\right.
$$

where $x_{p k}(\mathrm{k}=1,2,3)$ are the state variables of PI controllers in MSC control loops. $K_{p p k}$ and $K_{\text {pik }}(\mathrm{k}=1,2,3)$ are the proportional and integral parameters shown in Figure $1 \mathrm{a} . X_{* \text { ref }}$ denotes the reference value of variable $X_{*}$, and such denotations are also applied to other variables in the following content. 


\section{Modeling of DC-link}

The equation of the DC-link voltage is derived as

$$
C_{p} V_{p d c} \frac{d V_{p d c}}{d t}=P_{p s}-P_{p c}
$$

where $C_{p}$ is the capacitance, $V_{p d c}$ is DC voltage across the capacitor, $P_{p c}$ is the active power output and expressed as

$$
P_{p c}=V_{p c d} I_{p c d}+V_{p c q} I_{p c q}=V_{p d} I_{p c d}+V_{p q} I_{p c q}
$$

where $V_{p c k}$ and $I_{p c k}(\mathrm{k}=\mathrm{d}, \mathrm{q})$ are the direct and quadrature axis output voltage and output current of GSC, respectively. $V_{p d}$ and $V_{p q}$ are the direct and quadrature axis voltage at the point of common coupling (PCC), respectively.

The line voltage equations across the filter reactance $X_{p f}$ in Figure $1 \mathrm{~b}$ is expressed as

$$
\left\{\begin{array}{l}
\frac{d I_{p c d}}{d t}=\frac{\omega_{0}}{X_{p f}}\left(V_{p c d}-V_{p d}\right)+\omega_{0} I_{p c q} \\
\frac{d I_{p c q}}{d t}=\frac{\omega_{0}}{X_{p f}}\left(V_{p c q}-V_{p q}\right)-\omega_{0} I_{p c d}
\end{array}\right.
$$

4. Modeling of GSC

A standard GSC configuration is shown in Figure 1b, and the mathematic equations are derived as

$$
\left\{\begin{aligned}
\frac{d x_{p 4}}{d t} & =K_{p i 4}\left(V_{p d c}-V_{p d c r e f}\right) \\
\frac{d x_{p 5}}{d t} & =K_{p i 5}\left(I_{p c d r e f}-I_{p c d}\right) \\
\frac{d x_{p 6}}{d t} & =K_{p i 6}\left(Q_{p}-Q_{p r e f}\right) \\
\frac{d x_{p 7}}{d t} & =K_{p i 7}\left(I_{p c q r e f}-I_{p c q}\right)
\end{aligned}\right.
$$

where $x_{\mathrm{pk}}(\mathrm{k}=4,5,6,7)$ are the state variables of PI controllers in GSC control loops. $K_{p p k}$ and $K_{\text {pik }}(\mathrm{k}=4,5,6,7)$ are the proportional and integral parameters shown in Figure $1 \mathrm{~b}$. $Q_{p}$ is the reactive power output of GSC and expressed as

$$
Q_{p}=V_{p q} I_{p c d}-V_{p d} I_{p c q}
$$

5. Modeling of PLL

From the block diagram of SRF-PLL shown in Figure 2,

$$
\left\{\begin{array}{l}
\frac{d}{d x} x_{p l l}=K_{i p l l} V_{p q} \\
\frac{d}{d t} \theta_{p l l}=x_{p l l}+K_{p p l l} V_{p q}+\omega_{p l l r e f}
\end{array}\right.
$$

where $x_{p l l}$ and $\theta_{p l l}$ (i.e., phase angle) are the state variables of PLL dynamics, $\omega_{\text {pllref }}$ is the angular speed of PLL in rad/s, $K_{i p l l}$ and $K_{p p l l}$ are the integral and proportional parameters of the PLL controller, respectively.

By linearizing Equations (1)-(10) and combining them, the linearized state-space model of FCWG can be expressed as [3]

$$
\left\{\begin{array}{l}
\frac{\mathrm{d}}{\mathrm{dt}} \Delta \mathbf{X}_{\mathbf{w}}=\mathbf{A}_{\mathbf{w}} \Delta \mathbf{X}_{\mathbf{w}}+\mathbf{B}_{\mathbf{w}} \Delta \mathbf{V}_{\mathbf{w}} \\
\Delta \mathbf{I}_{\mathbf{w}}=\mathbf{C}_{\mathbf{w}} \Delta \mathbf{X}_{\mathbf{w}}
\end{array}\right.
$$

where $\Delta \mathbf{X}_{\mathbf{w}}$ denotes all the state variables of FCWG (i.e., the differential state variables in equations above); $\mathbf{A}_{\mathbf{w}}, \mathbf{B}_{\mathbf{w}}, \mathbf{C}_{\mathbf{w}}$ are the state-space matrices after integrating all linearized differential equations; $\Delta \mathbf{V}_{\mathbf{w}}$ and $\Delta \mathbf{I}_{\mathbf{w}}$ denote the voltage variation and current variation at the PCC of FCWG in the common $x-y$ coordinate. 


\subsection{State-Space Model of FCPV}

1. Modeling of PV generation unit

According to the voltage-current characteristic of FCPV, the relation between the output voltage and current of an FCPV is expressed as [27]

$$
V_{p v}=\frac{N_{s} n k T}{q} \ln \left(\frac{N_{p} I_{s c} I_{r} / 100-I_{p v}}{N_{p} I_{r}}+1\right)
$$

where $V_{p v}$ and $\mathrm{I}_{p v}$ are voltage and current output respectively; $T$ is the junction temperature, $k$ is Boltzmann's constant, $N_{s}$ and $N_{p}$ are the number of PV cells in series and parallel respectively, $q$ is the charge of electron, $n$ is the ideality factor, $I_{r}$ is the irradiance, $I_{s c}$ is the short-circuit current, $I_{0}$ is the saturation current.

Therefore, the output active power from an FCPV is expressed as

$$
P_{p v}=V_{p v} I_{p v}
$$

An inductance is used to limit the change of output current of the FCPV, and its dynamics can be derived as

$$
\frac{d I_{p v}}{d t}=\frac{\omega_{0}}{L_{d c}}\left(V_{p v}-V_{d c 1}\right)
$$

where $V_{d c 1}$ is the input DC voltage of DC/DC converter.

\section{Modeling of DC/DC converter}

The control structure of DC/DC converter is shown in Figure 4. The DC/DC converter control system consists of two control loops, i.e., an outer active power control loop and an inner current control loop.

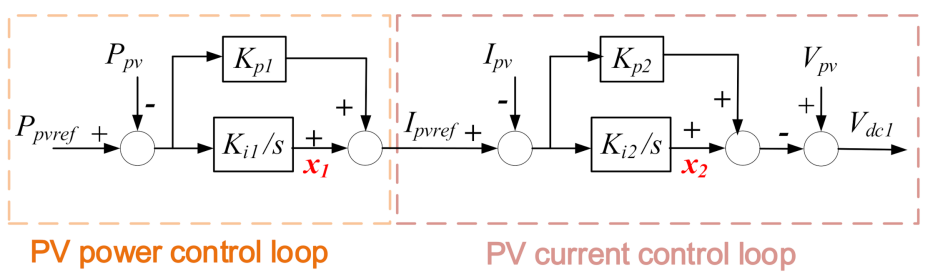

Figure 4. Control structure of DC/DC converter.

The dynamics of DC/DC converter control system can be written as

$$
\left\{\begin{array}{l}
\frac{d x_{1}}{d t}=K_{i 1}\left(P_{p v r e f}-P_{p v}\right) \\
I_{p v r e f}=x_{1}+K_{p 1}\left(P_{p v r e f}-P_{p v}\right) \\
\frac{d x_{2}}{d t}=K_{i 2}\left(I_{p v r e f}-I_{p v}\right) \\
V_{d c 1}=-x_{2}-K_{p 2}\left(I_{p v r e f}-I_{p v}\right)+V_{p v}
\end{array}\right.
$$

where $x_{1}$ and $x_{2}$ are the state variables of PI controllers, $K_{i 1}$ and $K_{p 1}$ are the integral and proportional parameters of the active power controller, $K_{i 2}$ and $K_{p 2}$ are the integral and proportional parameters of the current controller.

3. Modeling of DC-link

Ignoring the power loss of converters, the dynamic equation of the DC capacitor is expressed as

$$
C_{d c} V_{d c} \frac{d V_{d c}}{d t}=P_{p v}-P_{w}
$$


where $C_{d c}$ is DC capacitance, $V_{d c}$ is the voltage across the capacitor, $P_{p v}$ in the injected power from $P V$, and $P_{w}$ is the output active power in DC-link and defined as

$$
P_{w}=V_{w d} I_{w d}+V_{w q} I_{w q}
$$

where $I_{w d}$ and $I_{w q}$ are the direct and quadrature axis output current of GSC, respectively; $V_{w d}$ and $V_{w q}$ are direct and quadrature axis voltage of PCC, respectively.

The dynamics of the filter inductor are expressed as

$$
\left\{\begin{array}{l}
\frac{d I_{w d}}{d t}=\frac{\omega_{0}}{x_{w}}\left(V_{c d}-V_{w d}\right)+\omega_{0} I_{w q} \\
\frac{d I_{w q}}{d t}=\frac{\omega_{0}}{x_{w}}\left(V_{c q}-V_{w q}\right)-\omega_{0} I_{w d}
\end{array}\right.
$$

where $x_{\omega}$ is the inductance of the filter, $V_{c d}$ and $V_{c q}$ are direct and quadrature axis output voltage of DC/AC converter.

\section{Modeling of GSC}

The control structure of GSC is shown in Figure 5. GSC control system consists of four control loops: (1) the outer DC voltage control loop; (2) the inner direct axis current control loop; (3) the outer reactive power control loop; and (4) the inner quadrature axis current control loop.

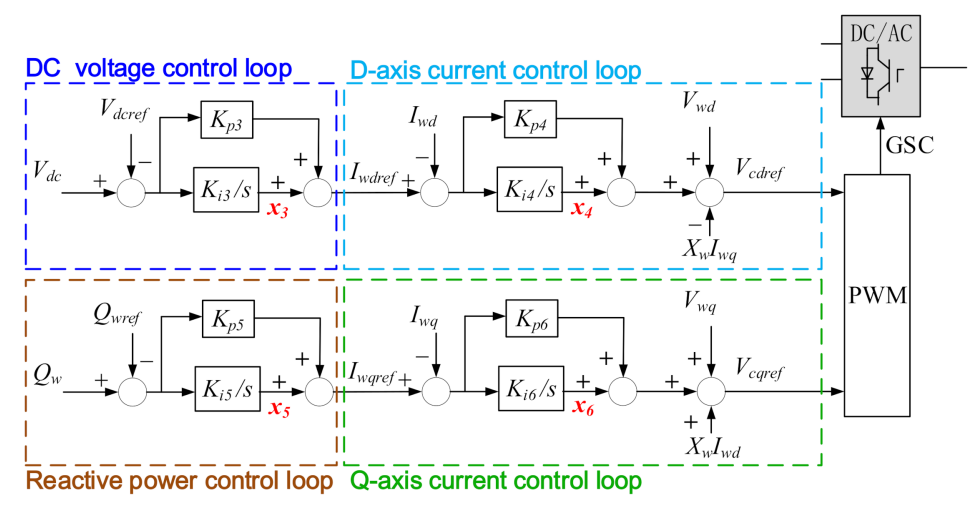

Figure 5. Control structure of GSC in FCPV.

The dynamics of GSC control system can be represented as

$$
\left\{\begin{array}{l}
\frac{d x_{3}}{d t}=K_{i 3}\left(V_{d c}-V_{d c r e f}\right) \\
I_{w d r e f}=K_{p 3}\left(V_{d c}-V_{d c r e f}\right)+x_{3} \\
\frac{d x_{4}}{d t}=K_{i 4}\left(I_{w d r e f}-I_{w d}\right) \\
V_{c d r e f}=K_{p 4}\left(I_{w d r e f}-I_{w d}\right)+x_{4}-X_{w} I_{w q}+V_{w d} \\
\frac{d x_{5}}{d t}=K_{i 5}\left(Q_{w}-Q_{w r e f}\right) \\
I_{w q r e f}=K_{p 5}\left(Q_{w}-Q_{w r e f}\right)+x_{5} \\
\frac{d x_{6}}{d t}=K_{i 6}\left(I_{w q r e f}-I_{w q}\right) \\
V_{c q r e f}=K_{6}\left(I_{w q r e f}-I_{w q}\right)+x_{p 6}+X_{w} I_{w d}+V_{w q}
\end{array}\right.
$$

where $x_{k}(\mathrm{k}=3,4,5,6)$ are the state variables of PI controllers, $K_{p k}$ and $K_{i k}(\mathrm{k}=3,4,5,6)$ are the parameters of corresponding PI controllers. $Q_{w}$ is the injected reactive power into power systems and expressed as

$$
Q_{w}=V_{w q} I_{w d}-V_{w d} I_{w q}
$$




\section{Modeling of SRF-PLL}

The same structure of the SRF-PLL shown in Figure 2 is applied in the FCPV and its dynamics are expressed as

$$
\left\{\begin{array}{l}
\frac{d}{d t} x_{p l l 2}=K_{i p l l 2} V_{w q} \\
\frac{d}{d t} \theta_{p l l 2}=x_{p l l 2}+K_{p p l l 2} V_{w q}+\omega_{p l l 2 r e f}
\end{array}\right.
$$

where all variables are similar to that of Equation (10). Accordingly, $\mathbf{X}_{*_{2}}$ is used in (21) corresponding to $\mathbf{X}_{*}$ in (10).

Therefore, by linearizing Equations (12)-(21), the linearized state-space model of FCPV is obtained as

$$
\left\{\begin{array}{l}
\frac{\mathrm{d}}{\mathrm{dt}} \Delta \mathbf{X}_{\mathbf{p v}}=\mathbf{A}_{\mathbf{p v}} \Delta \mathbf{X}_{\mathbf{p v}}+\mathbf{B}_{\mathbf{p v}} \Delta \mathbf{V}_{\mathbf{p v}} \\
\Delta \mathbf{I}_{\mathbf{p v}}=\mathbf{C}_{\mathbf{p v}} \Delta \mathbf{X}_{\mathbf{p v}}
\end{array}\right.
$$

where $\Delta \mathbf{X}_{\mathbf{p v}}$ denotes all state variables of FCPV; $\mathbf{A}_{\mathbf{p v}}, \mathbf{B}_{\mathbf{p v}}, \mathbf{C}_{\mathbf{p v}}$ are state-space matrices after integrating all the linearized differential equations. $\Delta \mathbf{V}_{\mathbf{p v}}$ and $\Delta \mathbf{I}_{\mathbf{p v}}$ denote the voltage variation and current variation at the PCC of FCPV in the common $x-y$ coordinate.

\subsection{Linearized Modeling of HRES System}

Based on the linearized state-space models of FCWG in (11) and FCPV in (22), the linearized state-space model of an HRES system can be represented as

$$
\left\{\begin{array}{l}
\frac{\mathrm{d}}{\mathrm{dt}} \Delta \mathbf{X}_{\mathrm{RES}}=\mathbf{A}_{\mathrm{RES}} \Delta \mathbf{X}_{\mathrm{RES}}+\mathbf{B}_{\mathrm{RES}} \Delta \mathbf{V}_{\mathbf{M}} \\
\Delta \mathbf{I}_{\mathbf{M}}=\mathbf{C}_{\mathrm{RES}} \Delta \mathbf{X}_{\mathrm{RES}}
\end{array}\right.
$$

where

$\Delta \mathbf{X}_{\mathrm{RES}}=\left[\Delta \mathbf{X}_{\mathrm{WG}}^{\mathrm{T}} \Delta \mathbf{X}_{\mathrm{PVG}}^{\mathrm{T}}\right]^{\mathrm{T}}, \Delta \mathbf{X}_{\mathrm{WG}}^{\mathrm{T}}=\left[\Delta \mathbf{X}_{\mathrm{w} 1}^{\mathrm{T}} \Delta \mathbf{X}_{\mathrm{w} 2}^{\mathrm{T}} \ldots \Delta \mathbf{X}_{\mathrm{wM}}^{\mathrm{T}}\right]^{\mathrm{T}}, \Delta \mathbf{X}_{\mathrm{PVG}}^{\mathrm{T}}=\left[\Delta \mathbf{X}_{\mathrm{p} 1}^{\mathrm{T}} \Delta \mathbf{X}_{\mathrm{pv} 2}^{\mathrm{T}} \ldots \Delta \mathbf{X}_{\mathrm{pvN}}^{\mathrm{T}}\right]^{\mathrm{T}}$, $\mathbf{A}_{\mathbf{R E S}}=\operatorname{diag}\left[\begin{array}{lll}\mathbf{A}_{\mathbf{W G}} & \mathbf{A}_{\mathbf{P V G}}\end{array}\right], \mathbf{A}_{\mathbf{W G}}=\operatorname{diag}\left[\begin{array}{lllll}\mathbf{A}_{\mathbf{w} 1} & \mathbf{A}_{\mathbf{w} 2} & \ldots & \mathbf{A}_{\mathbf{w M}}\end{array}\right], \mathbf{A}_{\mathbf{P V G}}=\operatorname{diag}\left[\begin{array}{lllll}\mathbf{A}_{\mathbf{p v} 1} & \mathbf{A}_{\mathbf{p v} 2} & \ldots & \mathbf{A}_{\mathbf{p v M}}\end{array}\right]$,

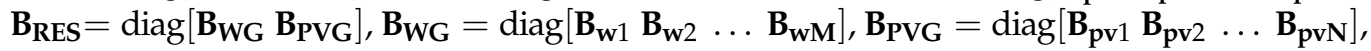
$\mathbf{C}_{\mathbf{R E S}}=\operatorname{diag}\left[\mathbf{C}_{\mathrm{WG}} \mathbf{C}_{\mathrm{PVG}}\right], \mathbf{C}_{\mathrm{WG}}=\operatorname{diag}\left[\mathbf{C}_{\mathbf{w} 1} \mathbf{C}_{\mathbf{w} 2} \ldots \mathbf{C}_{\mathbf{w M}}\right], \mathbf{C}_{\mathbf{P V G}}=\operatorname{diag}\left[\mathbf{C}_{\mathrm{pv} 1} \mathbf{C}_{\mathrm{pv} 2} \ldots \mathbf{C}_{\mathrm{pvN}}\right] ;$ $\mathbf{A}_{\mathbf{w x}}, \mathbf{B}_{\mathrm{wx}}, \mathbf{C}_{\mathrm{wx}}(\mathrm{x}=1,2, \ldots, \mathrm{M})$ are state matrices of the 1st to Mth FCWG, respectively; $\mathbf{A}_{\mathbf{p v x}}, \mathbf{B}_{\mathrm{pvx}}, \mathbf{C}_{\mathbf{p v x}}(\mathrm{x}=1,2, \ldots, \mathrm{N})$ are state matrices of the 1st to Nth FCPV, respectively; $\Delta \mathbf{X}_{\mathrm{wX}}^{\mathrm{T}}(\mathrm{x}=1,2, \ldots, \mathrm{M})$ and $\Delta \mathbf{X}_{\mathrm{pvx}}^{\mathrm{T}}(\mathrm{x}=1,2, \ldots, \mathrm{N})$ denote the vector of all state variables of FCWGs and FCPVs, respectively; $\Delta \mathbf{V}_{\mathbf{M}}$ and $\Delta \mathbf{I}_{\mathbf{M}}$ are the voltage variation and current variation of the HRES system at PCCs. diag[] denotes either a diagonal matrix or a block diagonal matrix.

\subsection{Entire Interconnected Power System}

In the open-loop power system, the HRES system is modeled as a constant power source. Assume that the state-space model for N SGs in the AC power system is expressed as

$$
\left\{\begin{array}{c}
\frac{\mathrm{d}}{\mathrm{dt}} \Delta \mathbf{X}_{\mathbf{g}}=\mathbf{A}_{\mathbf{g}} \Delta \mathbf{X}_{\mathbf{g}}+\mathbf{B}_{\mathbf{g}} \Delta \mathbf{V}_{\mathbf{g}} \\
\Delta \mathbf{I}_{\mathbf{g}}=\mathbf{C}_{\mathbf{g}} \Delta \mathbf{X}_{\mathbf{g}}+\mathbf{D}_{\mathbf{g}} \Delta \mathbf{V}_{\mathbf{g}}
\end{array}\right.
$$

where $\Delta \mathbf{X}_{\mathrm{g}}=\left[\Delta \mathbf{X}_{\mathrm{g} 1}^{\mathrm{T}} \Delta \mathbf{X}_{\mathrm{g} 2}^{\mathrm{T}} \ldots \Delta \mathbf{X}_{\mathrm{gN}}^{\mathrm{T}}\right]^{\mathrm{T}}, \Delta \mathbf{I}_{\mathrm{g}}=\left[\Delta \mathbf{I}_{\mathrm{g} 1}^{\mathrm{T}} \Delta \mathbf{I}_{\mathrm{g} 2}^{\mathrm{T}} \ldots \Delta \mathbf{I}_{\mathrm{gN}}^{\mathrm{T}}\right]^{\mathrm{T}}, \Delta \mathbf{V}_{\mathbf{g}}=\left[\Delta \mathbf{V}_{\mathrm{g} 1}^{\mathrm{T}} \Delta \mathbf{V}_{\mathrm{g} 2}^{\mathrm{T}} \ldots \Delta \mathbf{V}_{\mathrm{gN}}^{\mathrm{T}}\right]^{\mathrm{T}}$, $\mathbf{A}_{\mathbf{g}}=\operatorname{diag}\left[\begin{array}{llll}\mathbf{A}_{\mathrm{g} 1} & \mathbf{A}_{\mathrm{g} 2} \ldots & \mathbf{A}_{\mathrm{gN}}\end{array}\right], \quad \mathbf{B}_{\mathrm{g}}=\operatorname{diag}\left[\begin{array}{lllll}\mathbf{B}_{\mathrm{g} 1} & \mathbf{B}_{\mathrm{g} 2} \ldots & \mathbf{B}_{\mathrm{gN}}\end{array}\right], \quad \mathbf{C}_{\mathrm{g}}=\operatorname{diag}\left[\begin{array}{llll}\mathbf{C}_{\mathrm{g} 1} & \mathbf{C}_{\mathrm{g} 2} \ldots & \mathbf{C}_{\mathrm{gN}}\end{array}\right]$, $\mathbf{D}_{\mathbf{g}}=\operatorname{diag}\left[\begin{array}{lll}\mathbf{D}_{\mathbf{g} 1} & \mathbf{D}_{\mathbf{g} 2} \ldots & \mathbf{D}_{\mathbf{g N}}\end{array}\right], \Delta \mathbf{X}_{\mathbf{g j}}^{\mathrm{T}}(j=1,2, \ldots, \mathbf{N})$ denotes the vector of all state variables of the jth SG. $\Delta \mathbf{I}_{\mathrm{gj}}^{\mathrm{T}}$ and $\Delta \mathbf{V}_{\mathrm{gj}}^{\mathrm{T}}(\mathrm{j}=1,2, \ldots, \mathrm{N})$ are the current variation and voltage variation at connecting bus of jth SG. $\mathbf{A}_{\mathbf{g}}, \mathbf{B}_{\mathbf{g}}, \mathbf{C}_{\mathbf{g}}$, and $\mathbf{D}_{\mathbf{g j}}(j=1,2, \ldots, N)$ denote state-space matrices of the jth $\mathrm{SG}$.

The equation of the transmission network is expressed as

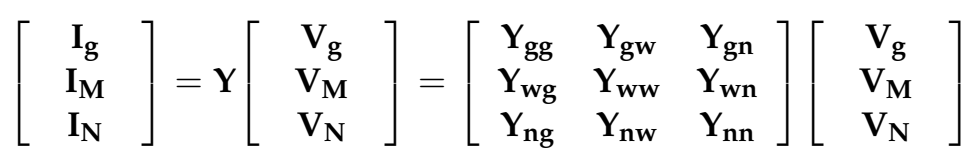


where $\mathbf{I}_{\mathbf{g}}$ and $\mathbf{V}_{\mathbf{g}}$ denote the current injection and voltage at the connecting buses of all SGs; $\mathbf{I}_{\mathbf{M}}$ and $\mathbf{V}_{\mathbf{M}}$ denote the current injection and voltage at PCCs of the HRES system; $\mathbf{I}_{\mathbf{N}}$ and $\mathbf{V}_{\mathbf{N}}$ denote the current injection and voltage at other buses in the network; $\mathbf{Y}$ denotes the admittance matrix.

From Equations (24) and (25), the open-loop power system can be derived as

$$
\left\{\begin{array}{l}
\frac{\mathrm{d}}{\mathrm{dt}} \Delta \mathbf{X}_{\mathbf{g}}=\mathbf{A}_{\mathbf{g s}} \Delta \mathbf{X}_{\mathbf{g}}+\mathbf{B}_{\mathrm{gs}} \Delta \mathbf{I}_{\mathbf{M}} \\
\Delta \mathbf{V}_{\mathbf{M}}=\mathbf{C}_{\mathbf{g s}} \Delta \mathbf{X}_{\mathbf{g}}+\mathbf{d}_{\mathbf{g s}} \Delta \mathbf{I}_{\mathbf{M}}
\end{array}\right.
$$

where

$$
\begin{aligned}
& A_{g s}=A_{g}+B_{g}\left(Y_{g g N}-Y_{g w N} Y_{w w N}^{-1} Y_{w g N}-D_{g}\right)^{-1} C_{g}, \\
& B_{g s}=-B_{g}\left(Y_{g g N}-Y_{g w N} Y_{w w N}^{-1} Y_{w g N}-D_{g}\right)^{-1} Y_{g w N} Y_{w w N}^{-1} \\
& C_{g s}=-Y_{w w N}^{-1} Y_{w g N}\left(Y_{g g N}-Y_{g w N} Y_{w w N}^{-1} Y_{w g N}-D_{g}\right)^{-1} C_{g}, \\
& d_{g s}=Y_{w w N}^{-1}+Y_{w w N}^{-1} Y_{w g N}\left(Y_{g g N}-Y_{g w N} Y_{w w N}^{-1} Y_{w g N}-D_{g}\right)^{-1} Y_{g w N} Y_{w w N}^{-1} \\
& Y_{g g N}=\left(Y_{g g}-Y_{g n} Y_{n n}^{-1} Y_{n g}\right), Y_{g w N}=Y_{g w}-Y_{g n} Y_{n n}^{-1} Y_{n w} \\
& Y_{w g N}=\left(Y_{w g}-Y_{w n} Y_{n n}^{-1} Y_{n g}\right), Y_{w w N}=\left(Y_{w w}-Y_{w n} Y_{n n}^{-1} Y_{n w}\right) .
\end{aligned}
$$

From Equations (23) and (26), the closed-loop interconnected model of the power system can be derived as

$$
\frac{\mathrm{d}}{\mathrm{dt}}\left[\begin{array}{c}
\Delta \mathbf{X}_{\mathrm{g}} \\
\Delta \mathbf{X}_{\mathrm{RES}}
\end{array}\right]=\left[\begin{array}{cc}
\mathbf{A}_{\mathrm{gS}} & \mathbf{B}_{\mathrm{gs}} \mathbf{C}_{\text {RES }} \\
\mathbf{B}_{\mathrm{RES}} \mathbf{C}_{\mathrm{gS}} & \mathbf{A}_{\mathrm{RES}}+\mathbf{B}_{\mathrm{RES}} \mathbf{d}_{\mathrm{gs}} \mathbf{C}_{\mathrm{RES}}
\end{array}\right]\left[\begin{array}{c}
\Delta \mathbf{X}_{\mathrm{g}} \\
\Delta \mathbf{X}_{\mathrm{RES}}
\end{array}\right]
$$

\section{Methodology of Optimization Strategy}

According to the modal superposition theory in [20], modal interaction can be categorized into three types: (1) weak interaction which indicates the HRES system interacts very slightly with the AC power system and thus the interaction effect can be ignored while studying converter-driven stability; (2) modal resonance that drives adjacent oscillation modes to move against each and thus impairs the system damping and threatens converter-driven stability; and (3) modal counteraction that implies that the HRES system interacts positively with AC power system and improves the system damping. It is worth mentioning that the negative modal resonance will jeopardize converter-driven stability and thus should be avoided, while the beneficial modal counteraction would be a favorable choice when integrating an HRES system.

To facilitate the positive interaction between the HRES system and the AC power system, an eigenvalue shift index (ESI) is utilized to quantitively evaluate the effect of modal interaction on the critical EOM. Denote $\lambda_{\text {olsysi }}=\sigma \pm j \omega$ as the ith oscillation mode of the open-loop power system, $\lambda_{\text {clsysi }}=\hat{\sigma} \pm j \hat{\omega}$ as the $i$ th oscillation mode of the closedloop system. Hence, the modal interaction effect of the newly introduced HRES system is evaluated by ESI $=\Delta \lambda_{s y s}=\lambda_{\text {clsysi }}-\lambda_{\text {olsysi }}$. According to the open-loop and closed-loop models in Section 3.4, modal analysis can be applied and thus provide a quantitative calculation for ESI.

If the real part of ESI, i.e., $\operatorname{Re}(\mathrm{ESI})<0$, demonstrates that the modal interaction is beneficial for the converter-driven stability. However, if $\operatorname{Re}(\mathrm{ESI})>0$, a detrimental impact regarding modal interaction is induced and deteriorates the converter-driven stability. To tackle this negative impact, a modal interaction optimization strategy can be implemented by tuning the control parameters of the HRES system. The optimization objective, as expressed in (28) is to obtain the largest modal shift in critical EOM towards the left half complex plane.

$$
\text { Minimize } \operatorname{Re}(E S I)=\operatorname{real}\left(\Delta \lambda_{\text {sysi }}\right)
$$

where $\Delta \lambda_{\text {sysi }}$ represents the eigenvalue shifts of interactive EOMs. 
It should be pointed out that the modal interaction optimization should not sacrifice the dynamics of the HRES system at an unacceptable level. Since parameter tuning is performed in HRES controllers, it is necessary and feasible to guarantee a sufficient damping margin for the HRES system. In this study, the PLLs of FCWG and FCPV were identified to be key components to optimize the multi-modal interaction with two EOMs of AC power system, and thus this can be regarded as a multi-modal multi-parametric optimization problem. The multi-objective particle swarm optimization (MOPSO) algorithm was applied to tune the parameters of key controllers.

\section{Case Study}

\subsection{Introduction of Test Power System}

An IEEE 16-machine 68-bus system [28] with an HRES system connected at bus 8 is illustrated in Figure 6. The HRES system consists of an FCWG-based wind farm and an FCPV-based solar energy farm. To emulate different penetration levels, four operating condition cases were thoroughly studied to uncover the impact on converterdriven stability regarding the modal interaction between the HRES system and AC power system. Denote the active power outputs (in per unit with base $S_{b}=100 \mathrm{MVA}$ ) of FCWG and FCPV as $P_{W G}$ and $P_{P V}$ respectively. The four operating condition cases were: (1) Case 1: $P_{W G}=0, P_{P V}=0$; (2) Case 2: $P_{W G}=0.5$ p.u., $P_{P V}=0.5$ p.u.; (3) Case 3: $P_{W G}=1.0$ p.u., $P_{P V}=1.5$ p.u.; and (4) Case $4: P_{W G}=2.0$ p.u., $P_{P V}=2.5$ p.u. Both FCWG and FCPV adopted reactive power control with constant power factor of 0.98 . The test system was built on the Matlab R2020b platform via M-language programming.

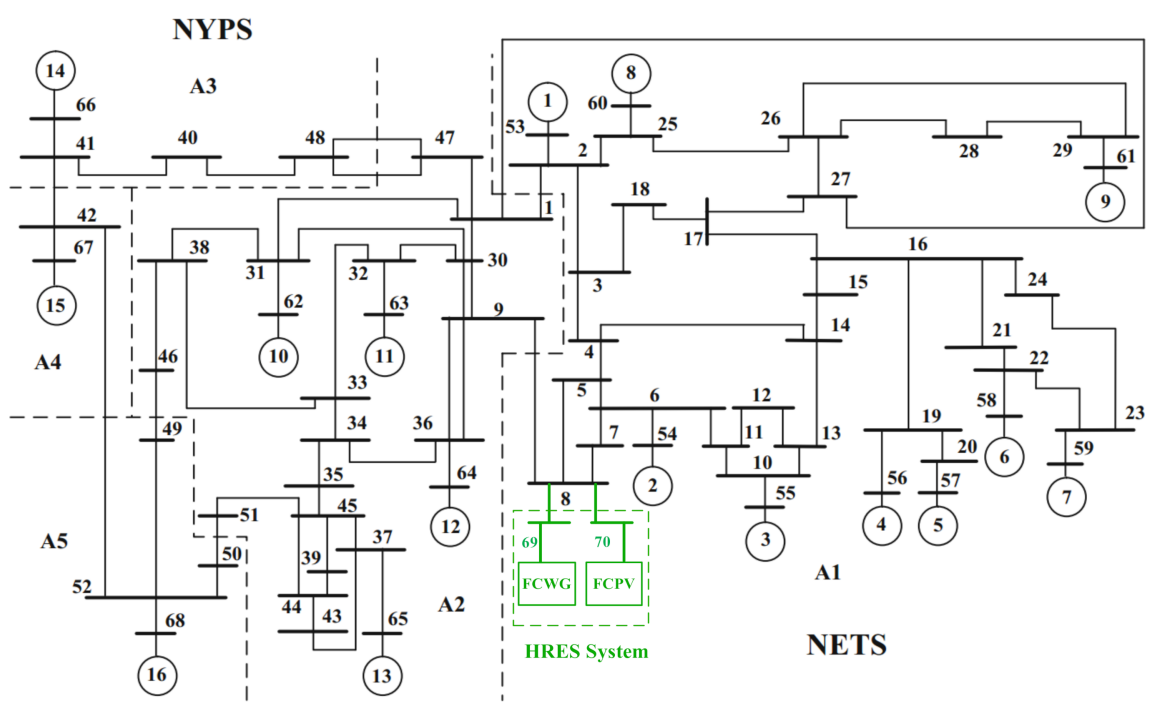

Figure 6. Configuration of test IEEE power system integrated with an HRES system.

The modal interaction can be either weak or strong. In a weak interaction case, the interaction impact can be ignored and the HRES system can be regarded as a constant power source. However, in strong interaction cases, the modal interaction between the HRES system and AC power system might have a considerable impact on the converter-driven stability and thus will be carefully addressed in the following analyses.

To begin with, open-loop modal analyses of the examined power systems with different wind power and PV solar energy penetration level were performed. Then, closed-loop modal resonance analyses of the closed-loop system and time-domain simulations were performed as supplementary verification. To mitigate the detrimental effect caused by strong modal interaction (i.e., modal resonance), the modal interaction optimization strategy was further adopted as well. 


\subsection{Converter-Driven Stablity Analyses Considering Different Renewable Energy Penetration Levels}

Based on the linearized state-space model the state matrix in Equation (26), the critical EOM of the open-loop power systems can be calculated, as exhibited in Table 1. The open-loop critical EOM of the power systems examined was slightly affected by different constant injected active power after ignoring the modal interaction of FCWG and FCPV. Since the open-loop EOM is mainly determined by the electromechanical dynamics of SGs in the AC power system, it had a relatively stable oscillation frequency $(0.57 \mathrm{~Hz}-0.60 \mathrm{~Hz})$ with a damping ratio (DR) around $2.9 \%$. This was also confirmed by the electromechanical loop correlation ratio (ELCR) [12], which stayed at a very high level (36.80-37.50).

Table 1. Open-loop modal analysis regarding different renewable energy penetration levels.

\begin{tabular}{ccccc}
\hline $\begin{array}{c}\text { Active Power from FCWG and } \\
\text { FCPV (p.u.) }\end{array}$ & Open-Loop Critical EOM $\left(\boldsymbol{\lambda}_{\text {opsys }}\right)$ & Frequency (Hz) & DR & ELCR \\
\hline Case 1 $(0,0)$ & $-0.1064 \pm 3.6391 \mathrm{i}$ & 0.5792 & $2.92 \%$ & 36.80 \\
Case 2 $(0.5,0.5)$ & $-0.1067 \pm 3.6694 \mathrm{i}$ & 0.5840 & $2.91 \%$ & 36.88 \\
Case 3 $(1.0,1.5)$ & $-0.1068 \pm 3.7158 \mathrm{i}$ & 0.5914 & $2.87 \%$ & 37.08 \\
Case 4 $(2.0,2.5)$ & $-0.1060 \pm 3.7790 \mathrm{i}$ & 0.6014 & $2.80 \%$ & 37.50 \\
\hline
\end{tabular}

As shown in Table 2, when the renewable energy penetration level grew higher, the modal interaction became stronger. This is because the modal interaction between the HRES system and AC power system is negative (viz., modal resonance), and such interaction effect is further magnified in the cases with large active power output from the HRES system [20]. The decrease in DR manifests that the closed-loop critical EOM becomes weaker and less stable. For instance, in Case 4, the closed-loop critical EOM had a negative DR $(-\mathbf{0 . 7 2} \%)$, which means that the system lost its converter-driven stability and became unstable.

Table 2. Closed-loop modal analysis regarding different renewable energy penetration levels.

\begin{tabular}{ccccc}
\hline $\begin{array}{c}\text { Active power from FCWG and } \\
\text { FCPV (p.u.) }\end{array}$ & Closed-Loop Critical EOM $\lambda_{\text {clsys }}$ & Frequency (Hz) & DR & ELCR \\
\hline Case 1 $(0,0)$ & $-0.1064 \pm 3.6391 \mathrm{i}$ & 0.5792 & $2.92 \%$ & 36.80 \\
Case 2 $(0.5,0.5)$ & $-0.0527 \pm 3.6022 \mathrm{i}$ & 0.5733 & $1.46 \%$ & 2.9990 \\
Case $3(1.0,1.5)$ & $-0.0105 \pm 3.5506 \mathrm{i}$ & 0.5651 & $0.30 \%$ & 1.8867 \\
Case 4 $(2.0,2.5)$ & $0.0251 \pm 3.4917 \mathrm{i}$ & 0.5557 & $-0.72 \%$ & 1.4929 \\
\hline
\end{tabular}

Participation factor analysis in Figure 7 illustrates that, in Case 1, since the active power of FCWG and FCPV were zero, they did not participate in the closed-loop critical EOM, and thus it stayed in the same position of the open-loop critical EOM. However, when the active power outputs of FCWG and FCPV increased, they began to actively interact with SGs and even became the dominant power sources (as shown in Case 3 and Case 4). A few very interesting and important findings are observed below. When we compare the active power outputs, although the total active power output of the HRES system (2.0 p.u. in Case 3 and 4.5 p.u. in Case 4 respectively) was less than one-tenth of the total active power of 13th and 15th SGs (45.91 p.u.), the HRES system had much larger participation factors for the critical EOM than that of the 13th and 15th SGs. It is also noteworthy that ELCR in Table 2 decreased significantly, which further indicates this critical EOM became less relevant to the electromechanical dynamics among SGs.

Through participation factor evaluation, it was also revealed that the closed-loop critical EOMs with oscillation frequencies between $0.55 \mathrm{~Hz}$ and $0.58 \mathrm{~Hz}$ were mainly dominated by PLL dynamics of the HRES system and electromechanical dynamics of the AC power system, respectively. Specifically, state variables related to PLL dynamics (i.e., $\Delta x_{\text {pll }}$, $\left.\Delta \theta_{p l l}, \Delta x_{p l l 2}, \Delta \theta_{p l l 2}\right)$ and SG electromechanical dynamics (i.e., $\left.\Delta \omega_{K}, \Delta \delta_{K},(K=1,2, \ldots, 16)\right)$ were the most active components in these closed-loop EOMs. 

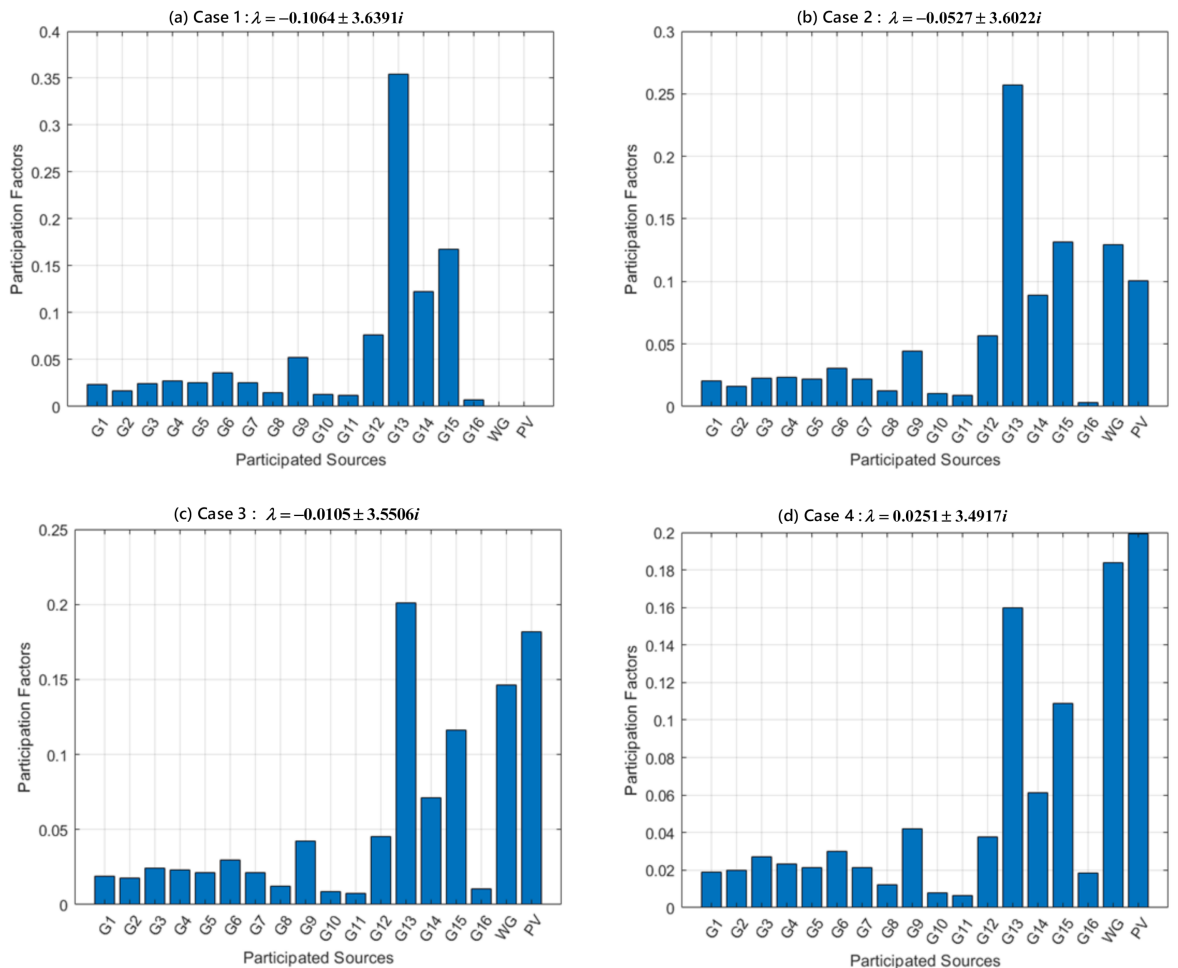

Figure 7. Participation factor analysis for closed-loop critical EOM $(0.55 \mathrm{~Hz}-0.58 \mathrm{~Hz})$. (a) Case 1; (b) Case 2; (c) Case 3; (d) Case 4.

It is worth mentioning that the integration of the HRES system not only affected the critical EOM, but also interacted with other EOMs with different intensity levels. Here we demonstrate the participation of HRES in another local EOM $(0.65 \mathrm{~Hz} \sim 0.67 \mathrm{~Hz}$, mainly dominated by 14th and 16th SGs and denoted as EOM2) in Figure 8. In Case 4, the HRES system had a total participation factor larger than 0.2, and both FCWG and FCPV participated. It can be seen that the DR of EOM2 increased from $2.22 \%$ to $3.60 \%$. In other words, the modal interaction effect on EOM2 was positive. Moreover, despite the large geographical/electrical distance between the HRES system and SGs (14th and 16th), great attention should be paid to their modal interaction, especially in the detrimental modal resonance cases. This also proves that the HRES dynamics can penetrate deeply into the local electromechanical dynamics. It further indicates that the interaction between the HRES system and AC power system can be multi-modal. In this study, two HRES oscillation modes and two EOMs were representative and involved in the modal interaction process.

Modal interaction also affects the oscillation modes of the HRES system. In this work, we investigated two critical oscillation modes of the HRES system, i.e., one from FCWG and the other from FCPV, which are denoted as FOM and POM respectively. It should be pointed out that there are 7 pairs of conjugated oscillation modes in FCWG and 5 pairs in FCPV. Only the two most active oscillation modes are listed and analyzed, and the other modes have a very limited interaction with the electromechanical dynamics and thus can be ignored in this study. The closed-loop modal analysis of the HRES system is presented in Table 3 (FCWG) and Table 4 (FCPV). The results of open-loop modal analysis are not listed separately here since they are almost the same as those of Case 1 in the closed-loop modal analysis. This is because the modal interaction impact is not considered in the open-loop modal analysis, and the power flow impact on modal shifts is minimal unless significant changes occur in the AC power system, as demonstrated in Table 1. 

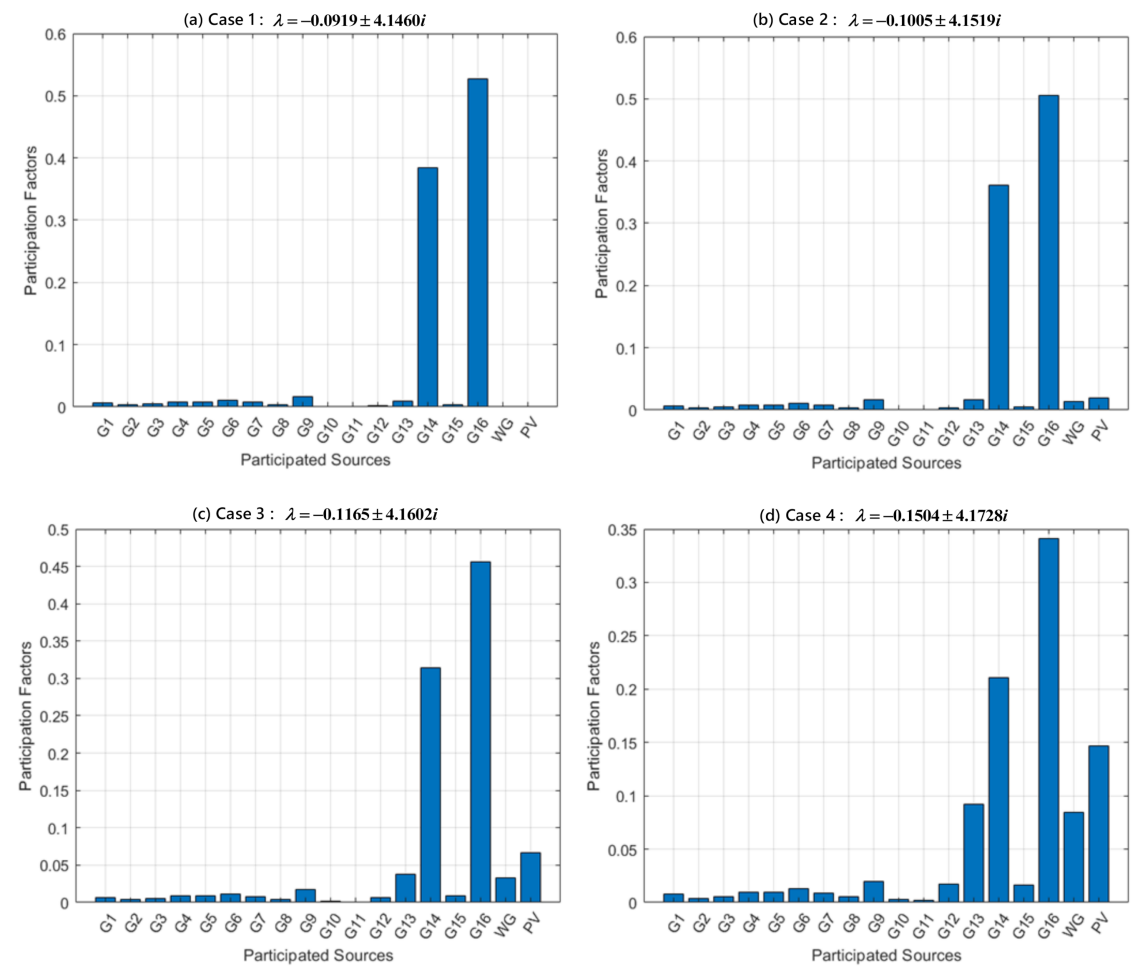

Figure 8. Participation factor analysis for closed-loop EOM2 (0.65 Hz 0.67 Hz). (a) Case 1; (b) Case 2; (c) Case 3; (d) Case 4 .

Table 3. Closed-loop modal analysis of FOM (wind power).

\begin{tabular}{ccccc}
\hline $\begin{array}{c}\text { Active Power from } \\
\text { FCWG (p.u.). }\end{array}$ & Closed-Loop FOM $\lambda_{\text {clwo }}$ & $\begin{array}{c}\text { Frequency } \\
\mathbf{( H z )}\end{array}$ & DR & ELCR \\
\hline Case 1 (0) & $-0.0702 \pm 3.7456 \mathrm{i}$ & 0.5961 & $1.87 \%$ & 0 \\
Case 2 (0.5) & $-0.0812 \pm 3.8706 \mathrm{i}$ & 0.6160 & $2.10 \%$ & 0.0482 \\
Case 3 (1.0) & $-0.0783 \pm 3.9024 \mathrm{i}$ & 0.6211 & $2.01 \%$ & 0.0187 \\
Case 4 (2.0) & $-0.0786 \pm 3.9446 \mathrm{i}$ & 0.6278 & $1.99 \%$ & 0.0122 \\
\hline
\end{tabular}

Table 4. Closed-loop modal analysis of POM (PV).

\begin{tabular}{ccccc}
\hline $\begin{array}{c}\text { Active Power from } \\
\text { FCPV (p.u.) }\end{array}$ & Closed-Loop POM $\lambda_{\text {clpv }}$ & $\begin{array}{c}\text { Frequency } \\
\mathbf{( H z )}\end{array}$ & DR & ELCR \\
\hline Case 1 (0) & $-0.0748 \pm 3.8683 \mathrm{i}$ & 0.6157 & $1.93 \%$ & 0 \\
\hline Case 2 (0.5) & $-0.1083 \pm 3.9019 \mathrm{i}$ & 0.6210 & $2.77 \%$ & 0.2868 \\
\hline Case 3 (1.5) & $-0.1327 \pm 3.9710 \mathrm{i}$ & 0.6320 & $3.34 \%$ & 0.6820 \\
\hline Case 4 (2.5) & $-0.1297 \pm 4.0445 \mathrm{i}$ & 0.6437 & $3.21 \%$ & $\mathbf{1 . 4 6 5 1}$ \\
\hline
\end{tabular}

In can be seen in Tables 3 and 4 that both closed-loop FOM and closed-loop POM were improved with damping increase. Meanwhile, the corresponding oscillation frequencies also increased slightly. It is important to highlight that, in Case 4, the closed-loop POM has a large ELCR (1.4651), which is greater than 1. Thus, this POM can be recognized as an EOM, and its participation factor analysis is exhibited in Figure 9. Five SGs (12th-16th SGs) participate actively in this POM. Moreover, FCWG also has a large participation (larger than 0.1) in this POM, which indicates that FCWG and FCPV might interact with each other and affect their own dynamics. 


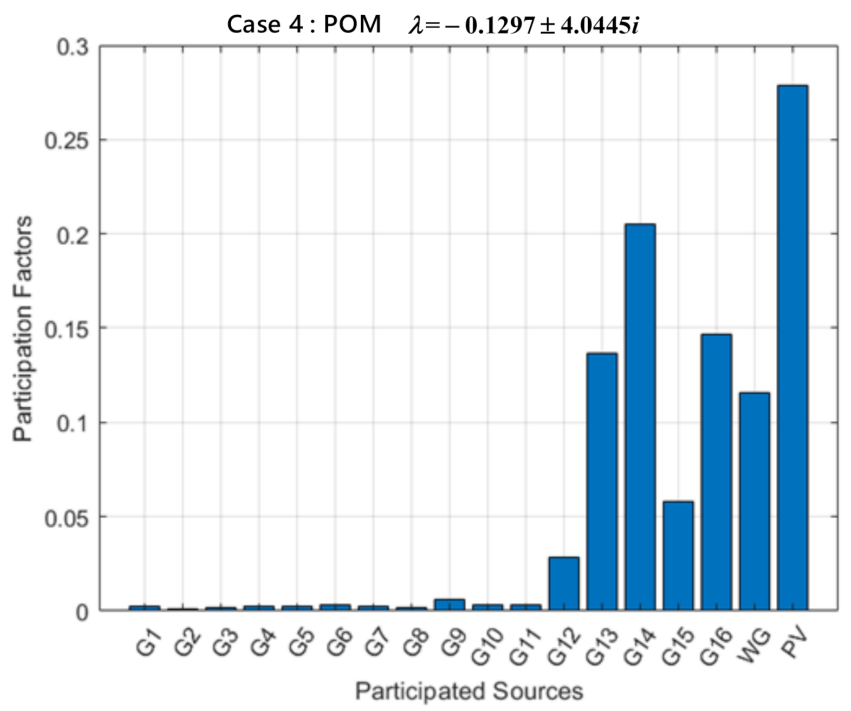

Figure 9. Participation factor analysis for closed-loop POM in Case 4.

To demonstrate the modal interaction effect discussed above, the modal shifts regarding four main interactive oscillation modes (i.e., Critical EOM, EOM2, FOM, and POM) from their open-loop location to closed-loop location are illustrated in Figure 10. $\lambda_{\text {clsysk }}, \lambda_{\text {clsys } 2 k}, \lambda_{\text {clwgk }}$, and $\lambda_{\text {clpvk }}$ (case number $k=1,2,3,4$ ) denote the eigenvalues of four closed-loop oscillation modes in four cases, respectively. It should be noted that the open-loop location of the oscillation modes in all four cases was almost of the same as their closed-loop location of Case 1 (i.e., $\lambda_{\text {clsys } 1}, \lambda_{\text {clsys } 21}, \lambda_{\text {clwg } 1}$, and $\lambda_{\text {clpv } 1}$ ). This phenomenon can be evidenced the information in Table 1 , as well as the nature of PLL oscillation modes of FCWG and FCPV. Hence, for better readability, the open-loop oscillation modes are not presented in Figure 10, and instead $\lambda_{\text {clsys } 1}, \lambda_{\text {clsys21 }}, \lambda_{\text {clw } 11}$, and $\lambda_{\text {clpv1 } 1}$ can be treated as the starting points of each modal shift. In other words, the modal shift of each oscillation mode due to the interaction is the eigenvalue movement from its starting point to the closed-loop oscillation mode. It is easy to conclude from Figure 10 that although EOM2 and POM were meliorated, the critical EOM was impaired and gradually shifted towards the unstable right half-plane.

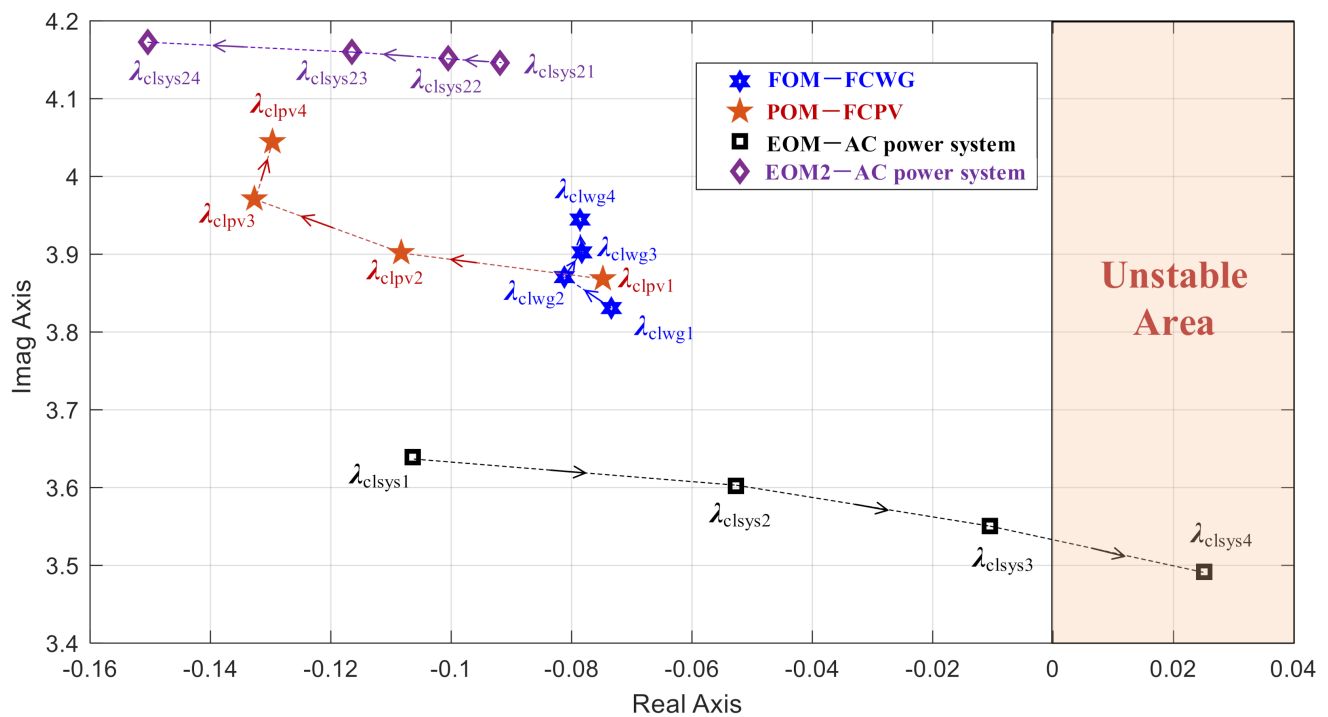

Figure 10. Modal shifts due to modal interaction in four cases. 
To verify modal analysis results and further illustrate converter-driven stability visually, time-domain simulations were carried out. The simulation conditions were set as: a 5\% step increase in the mechanical power of 1st SG occurs and subsequently drops to the original value after $100 \mathrm{~ms}$. From the participation factor analysis in Figure 7, 13th and 15th SGs had the largest and second-largest participation factors in the AC power system, respectively. Hence, the rotor speed difference between 13th and 15th SG and active power of 15th SG were demonstrated. The DRs in Table 2 are also marked in the simulation results.

From Figures 11 and 12, modal interaction between the HRES system and AC power system had a negative impact on the closed-loop critical EOM. Especially in Case 4, the 13th and 15th SGs lost synchronism and the active power output of 15th SG became unstable. Therefore, the simulation results are consistent with the modal analysis above.

\subsection{Modal Interaction Optimization to Enhance Converter-Driven Stability}

A modal interaction optimization strategy was implemented to mitigate the detrimental effect of modal resonance and enhance the converter-driven stability. By tuning parameters of FCWG and FCPV, the open-loop critical FOM and POM can not only be relocated but also induce beneficial modal interactions with the AC power system. As a result, the strong multi-modal resonance was eliminated. In this study, PLL control parameters of FCWG and FCPV were identified as pivotal parameters related to strong modal resonance. Therefore, the modal interaction optimization strategy was performed to appropriately adjust these parameters. The modified parameters and corresponding modal analysis results are listed in Table 5. Compared with the original power system with inadequate damping, the closed-loop critical EOM after optimization gains significantly improved damping, and hence better converter-driven stability.
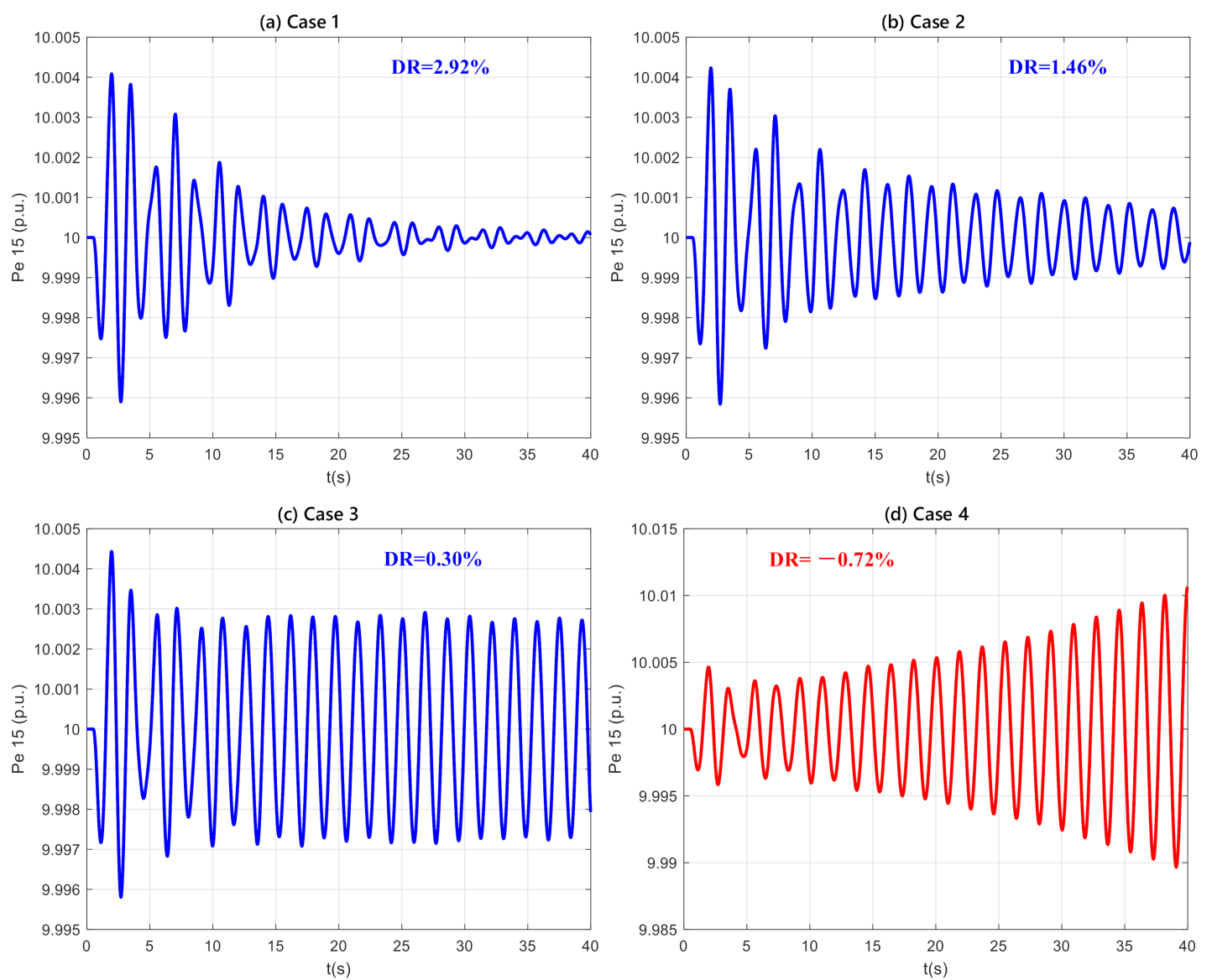

Figure 11. Rotor speed difference between 13th SG and 15th SG in four Cases. (a) Case 1; (b) Case 2; (c) Case 3; (d) Case 4. 

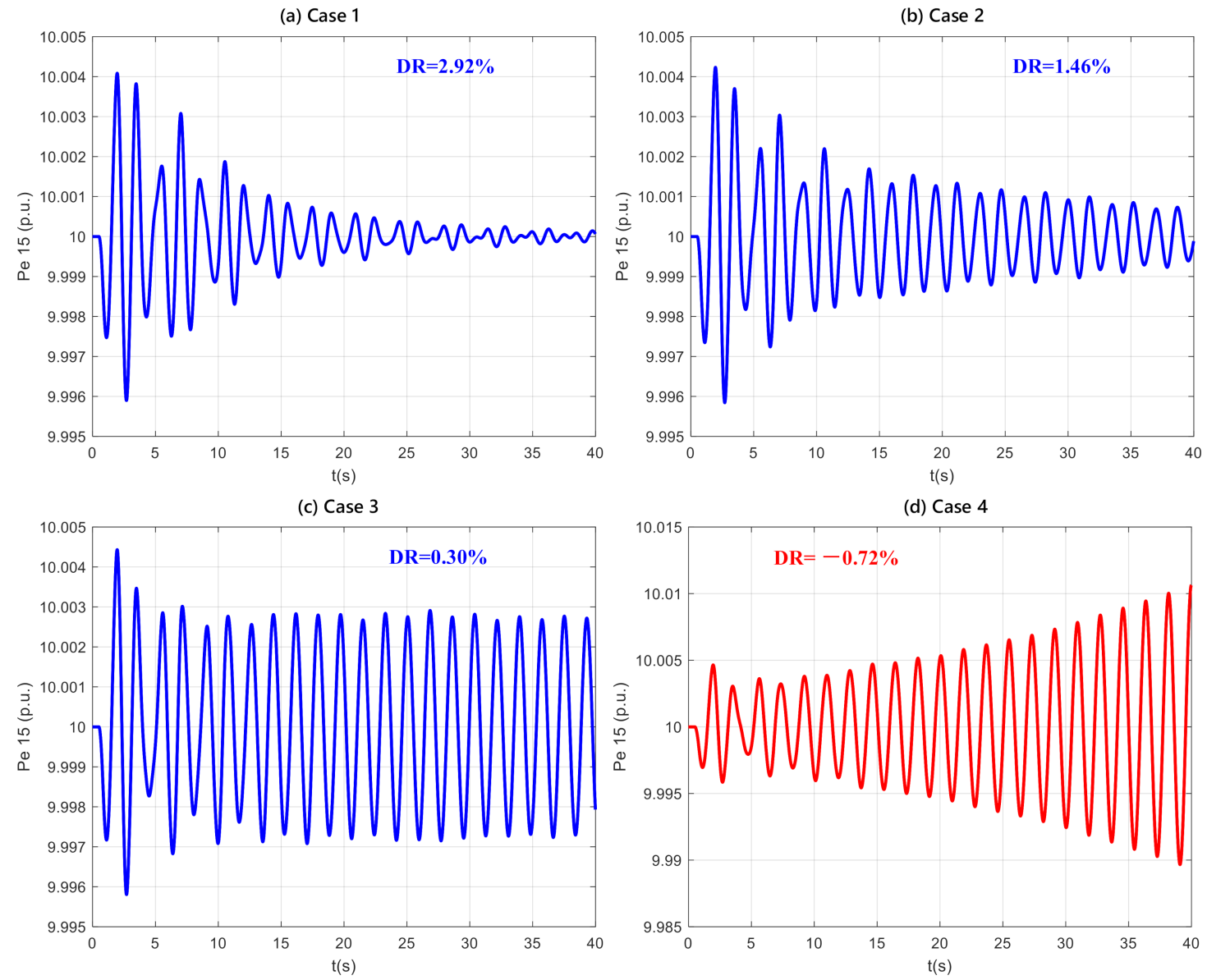

Figure 12. Active power output of 15th SG in four Cases. (a) Case 1; (b) Case 2; (c) Case 3; (d) Case 4.

Table 5. Parameter optimization of FCWG and FCPV.

\begin{tabular}{ccc}
\hline Type & Original Control Parameters & Optimized Control Parameters \\
\hline PLL control parameters of FCWG & $K_{p w g}=0.15, K_{i w g}=15$, & $K_{p w g}=0.5631, K_{i w g}=11.4376$ \\
PLL control parameters of FCPV & $K_{p p v}=0.16, K_{i p v}=16$ & $K_{p p v}=0.3487, K_{i p v}=16.3764$ \\
Open-loop critical FOM & $-0.0776 \pm 3.9385 \mathrm{i}(0.6268 \mathrm{~Hz}, 1.97 \%)$ & $-0.2731 \pm 3.3194 \mathrm{i}$ \\
(Freq., DR) & $-0.0786 \pm 3.9446 \mathrm{i}$ & $(0.5283 \mathrm{~Hz}, 8.20 \%)$ \\
Closed-loop critical FOM & $(0.6278 \mathrm{~Hz}, 1.99 \%)$ & $-0.1388 \pm 3.2740 \mathrm{i}$ \\
(Freq., DR) & $-0.0792 \pm 3.9783 \mathrm{i}$ & $(0.5211 \mathrm{~Hz}, 4.23 \%)$ \\
Open-loop critical POM & $(0.6332 \mathrm{~Hz}, 1.99 \%)$ & $-0.1725 \pm 4.0220 \mathrm{i}$ \\
(Freq., DR) & $-0.1297 \pm 4.0445 \mathrm{i}$ & $(0.6401 \mathrm{~Hz}, 4.29 \%)$ \\
Closed-loop critical POM & $(0.6437 \mathrm{~Hz}, 3.21 \%)$ & $-0.1907 \pm 4.1088 \mathrm{i}$ \\
(Freq., DR) & $-0.1060 \pm 3.7790 \mathrm{i}$ & $(0.6539 \mathrm{~Hz}, 4.64 \%)$ \\
Open-loop critical EOM & $(0.6024 \mathrm{~Hz}, 2.80 \%)$ & $-0.1060 \pm 3.7790 \mathrm{i}$ \\
(Freq., DR) & $0.0251 \pm 3.4917 \mathrm{i}$ & $(0.6024 \mathrm{~Hz}, 2.80 \%)$ \\
Closed-loop critical EOM & $(0.5557 \mathrm{~Hz},-\mathbf{0 . 7 2} \%)$ & $-0.1647 \pm 3.5897 \mathrm{i}$ \\
(Freq., DR) & $-0.0934 \pm 4.1669 \mathrm{i}$ & $(0.5713 \mathrm{~Hz}, 4.58 \%)$ \\
Open-loop EOM2 & $(0.6632 \mathrm{~Hz}, 2.24 \%)$ & $-0.0934 \pm 4.1669 \mathrm{i}$ \\
(Freq., DR) & $-0.1504 \pm 4.1728 \mathrm{i}$ & $(0.6632 \mathrm{~Hz}, 2.24 \%)$ \\
Closed-loop EOM2 & $(0.6641 \mathrm{~Hz}, 3.60 \%)$ & $-0.1323 \pm 4.1232 \mathrm{i}$ \\
(Freq., DR) & & $(0.6562 \mathrm{~Hz}, 3.21 \%)$ \\
\hline
\end{tabular}

The modal shifts of system EOMs are also presented in Figure 13. Compared with the original system, in the optimized system, the closed-loop critical EOM moves towards the left half complex plane. Its DR increases from $-\mathbf{0 . 7 2} \%$ to $\mathbf{4 . 5 8 \%}$, which is quite a prominent improvement. It should also be highlighted that such a change is only brought by the 
parameter tuning with the optimization strategy, while the operating condition remains the same. From the perspective of system operators, this method is very cost-effective since no additional control or devices involved.

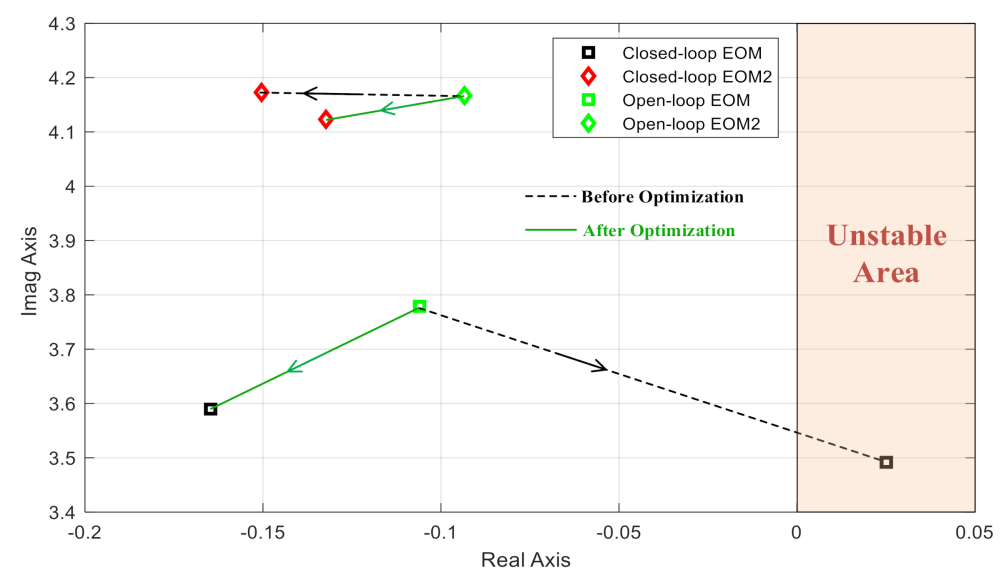

Figure 13. Modal shifts of system EOMs with/without optimization strategy.

The rotor speed difference between 13th and 15th SGs and active power of 15th SG are compared in Figure 14. The dynamic performance of the modified interconnected system was greatly improved. These results are in accordance with the DR indication of the closed-loop critical EOM in Table 5. Therefore, the implemented multi-modal and multi-parametric optimization strategy was found to be effective for reinforcing the converter-driven stability.
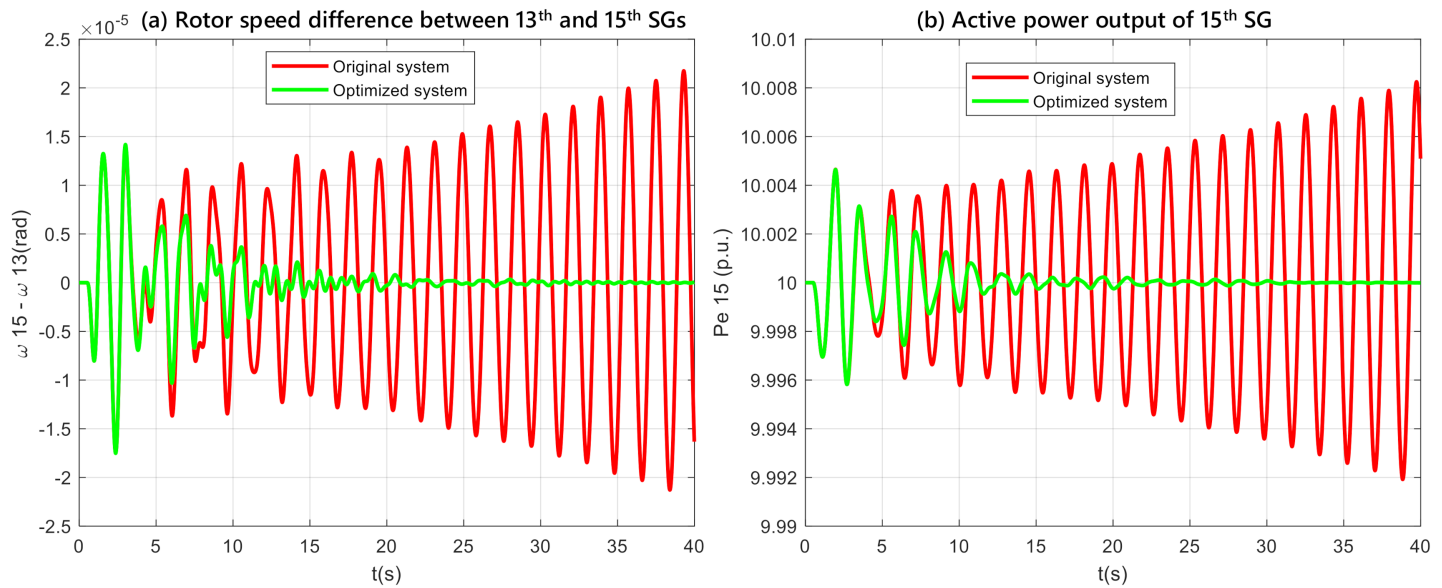

Figure 14. Comparison of simulation results in Case 4 before/after optimization. (a) Rotor speed difference between 13th and 15th SGs; (b) Active power output of 15th SG.

\section{Discussion}

In the above converter-driven stability analysis, various modal interaction conditions were thoroughly investigated by extensively evaluating the multi-modal interactions between the multi-RESs in the HRES system and AC power system. Several important findings are summarized as follows:

(1) From the open-loop modal analysis of the external AC power system, with the increasing active power injection of RESs, the open-loop critical EOM was very slightly affected. Such minor variations are mainly due to the power flow impact since the modal interaction of the HRES system was excluded in the open-loop analysis. 
(2) For the closed-loop modal analysis, the overall impact of HRES integration was evaluated, considering both the power flow impact and modal interactions. Strong modal interaction occurred when the open-loop critical FOM and POM were close to the open-loop EOMs in terms of oscillation frequency. This is a necessary condition to induce strong modal interaction.

(3) The multi-modal interaction involved oscillation modes of the multi-RESs in the HRES system and AC power system. The integration of the HRES system mainly affected two EOMs (i.e., one global critical EOM and one local EOM2). Strong modal interaction effects can be either positive or negative. For instance, in this study, the critical EOM deteriorated while the local EOM2 improved.

(4) The HRES system could participate more actively in the critical EOMs even at a much lower active power output compared to SGs. The HRES dynamics could penetrate deeply into the local electromechanical dynamics regardless of their geographical/electrical distance.

(5) The electromechanical dynamics of SGs might also participate in HRES oscillation modes (e.g., FOM and POM), which will lead to either positive or negative modal shifts in HRES oscillation modes. Their participation can also be quantified by ELCRs.

(6) Apart from the interaction between SGs and HRES, FCWG and FCPV inside the HRES system can also interact with each other and participate in each other's dynamics. Therefore, it is necessary to coordinate their interaction (e.g., parameter tuning) and avoid the interior modal resonance.

(7) An interesting phenomenon appears when electromechanical dynamics become quite active in an HRES oscillation mode, and thus this mode turns into an EOM. In Case 4, the closed-loop POM had an ELCR larger than 1, and hence it can be recognized as an EOM. Such phenomena usually happen at high HRES penetration levels.

(8) As a negative strong modal interaction, modal resonance might dramatically degrade system damping and thus should be circumvented. An effective countermeasure is to implement the modal interaction optimization strategy to properly modify the key parameters of HRES controllers. With the optimization strategy adopted, not only can the detrimental effect of modal resonance be alleviated, but also a positive modal interaction (i.e., modal counteraction) can be achieved.

\section{Conclusions}

In this work, an IEEE benchmark power system with an HRES system was investigated regarding the converter-driven stability. The converter-driven stability issues studied are in the form of modal interaction between two subsystems (i.e., HRES system and AC power system). Therefore, we elaborately examined the detrimental modal resonance conditions to reveal the mechanisms of potential converter-driven instability issues.

The integration of the HRES system not only affected the power flow but also interacted with the external AC power system. Particularly, when modal resonance happened, the critical system EOM was forced to move towards the right half complex plane, and thus threatened the converter-driven stability. An extreme condition with a multi-modal interaction case was examined to evaluate the consequence of strong modal resonance. The DR of closed-loop critical mode was greatly weakened or even became negative. As a result, oscillations once being aroused due to unexpected disturbances cannot be effectively suppressed and may further propagate in the external AC power system.

To prevent this adverse phenomenon, a modal interaction optimization strategy was implemented. The converter-driven stability was remarkably enhanced after relocating critical modes of the HRES system and meliorating its modal interaction with the AC power system. Consistent with modal analysis, time-domain simulation results substantiate that the groups of SGs can maintain synchronism in disturbance conditions after optimization in the HRES system.

Author Contributions: J.L. completed the methodology, theory analysis, validation, and wrote the paper. Y.Z. conducted data analysis, simulations, and validation. S.B. supervised the research through- 
out, acquired funding, coordinated the project, and revised \& edited the paper. U.K. conducted validation. All authors have read and agreed to the published version of the manuscript.

Funding: This research was funded by National Natural Science Foundation of China for the Research Project (52077188), Guangdong Science and Technology Department for the Research Project (2019A1515011226), Hong Kong Research Grant Council for the Research Projects (25203917), (15200418) and (15219619), and Department of Electrical Engineering, The Hong Kong Polytechnic University for the Start-up Fund Research Project (1-ZE68).

Institutional Review Board Statement: Not applicable.

Informed Consent Statement: Not applicable.

Data Availability Statement: Not applicable.

Conflicts of Interest: The authors declare no conflict of interest.

\section{References}

1. Quintero, J.; Vittal, V.; Heydt, G.T.; Zhang, H. The Impact of Increased Penetration of Converter Control-Based Generators on Power System Modes of Oscillation. IEEE Trans. Power Syst. 2014, 29, 2248-2256. [CrossRef]

2. Badal, F.R.; Das, P.; Sarker, S.K.; Das, S.K. A survey on control issues in renewable energy integration and microgrid. Prot. Control Mod. Power Syst. 2019, 4, 8. [CrossRef]

3. Luo, J.; Bu, S.; Zhu, J.; Chung, C.Y. Modal Shift Evaluation and Optimization for Resonance Mechanism Investigation and Mitigation of Power Systems Integrated With FCWG. IEEE Trans. Power Syst. 2020, 35, 4046-4055. [CrossRef]

4. Elliott, R.T.; Ellis, A.; Pourbeik, P.; Sanchez-Gasca, J.J.; Senthil, J.; Weber, J. Generic photovoltaic system models for WECC-A status report, 2015. In Proceedings of the IEEE Power \& Energy Society General Meeting, Denver, CO, USA, 26-30 July 2015; pp. 1-5.

5. Hatziargyriou, N.; Milanović, J.; Rahmann, C.; Ajjarapu, V.; Cañizares, C.; Erlich, I.; Hill, D.; Hiskens, I.; Kamwa, I.; Pal, B.S. Stability Definitions and Characterization of Dynamic Behavior in Systems with High Penetration of Power Electronic Interfaced Technologies; Technical Report No. PES-TR77; IEEE: Piscataway, NJ, USA, 2020; p. 42.

6. Adams, J.; Pappu, V.A.; Dixit, A. Ercot experience screening for Sub-Synchronous Control Interaction in the vicinity of series capacitor banks, 2012. In Proceedings of the IEEE Power and Energy Society General Meeting, San Diego, CA, USA, 22-26 July 2012; pp. 1-5.

7. Liu, H.; Xie, X.; Gao, X.; Liu, H.; Li, Y. Stability Analysis of SSR in Multiple Wind Farms Connected to Series-Compensated Systems Using Impedance Network Model. IEEE Trans. Power Syst. 2018, 33, 3118-3128. [CrossRef]

8. Luo, J.; Bu, S.; Chung, C.Y. Design and Comparison of Auxiliary Resonance controllers for Mitigating Modal Resonance of Power Systems Integrated with Wind Generation. IEEE Trans. Power Syst. 2021. [CrossRef]

9. Zhan, Y.; Xie, X.; Liu, H.; Liu, H.; Li, Y. Frequency-Domain Modal Analysis of the Oscillatory Stability of Power Systems With High-Penetration Renewables. IEEE Trans. Sustain. Energy 2019, 10, 1534-1543. [CrossRef]

10. Du, W.; Wang, H.F.; Bu, S. Small-Signal Stability Analysis of Power Systems Integrated with Variable Speed Wind Generators; Springer Nature: Cham, Switzerland, 2018.

11. Du, W.; Bi, J.; Wang, H. Damping Degradation of Power System Low-Frequency Electromechanical Oscillations Caused by Open-Loop Modal Resonance. IEEE Trans. Power Syst. 2018, 33, 5072-5081. [CrossRef]

12. Luo, J.; Bu, S.; Zhu, J. Transition from Electromechanical Dynamics to Quasi-Electromechanical Dynamics Caused by Participation of Full Converter-Based Wind Power Generation. Energies 2020, 13, 6270. [CrossRef]

13. Zhou, J.Z.; Ding, H.; Fan, S.; Zhang, Y.; Gole, A.M. Impact of Short-Circuit Ratio and Phase-Locked-Loop Parameters on the Small-Signal Behavior of a VSC-HVDC Converter. IEEE Trans. Power Deliv. 2014, 29, 2287-2296. [CrossRef]

14. Meegahapola, L.G.; Bu, S.; Wadduwage, D.P.; Chung, C.Y.; Yu, X. Review on Oscillatory Stability in Power Grids With Renewable Energy Sources: Monitoring, Analysis, and Control Using Synchrophasor Technology. IEEE Trans. Ind. Electron. 2021, 68, 519-531. [CrossRef]

15. Morató, J.; Knüppel, T.; Østergaard, J. Residue-Based Evaluation of the Use of Wind Power Plants With Full Converter Wind Turbines for Power Oscillation Damping Control. IEEE Trans. Sustain. Energy 2014, 5, 82-89. [CrossRef]

16. Knüppel, T.; Nielsen, J.N.; Jensen, K.H.; Dixon, A.; Østergaard, J. Power Oscillation Damping Capabilities of Wind Power Plant with Full Converter Wind Turbines Considering its Distributed and Modular Characteristics. IET Renew. Power Gener. 2013, 7, 431-442. [CrossRef]

17. Haque, M.E.; Negnevitsky, M.; Muttaqi, K.M. A Novel Control Strategy for a Variable Speed Wind Turbine with a Permanent Magnet Synchronous Generator, 2008. In Proceedings of the IEEE Industry Applications Society Annual Meeting, Edmonton, AB, Canada, 5-9 October 2008; pp. 1-8.

18. Jamehbozorg, A.; Radman, G. Small Signal Analysis of Power Systems with Wind and Energy Storage Units. IEEE Trans. Power Syst. 2015, 30, 298-305. [CrossRef] 
19. Luo, J.; Bu, S.; Zhu, J. A Novel PMU-based Adaptive Coordination Strategy to Mitigate Modal Resonance between Full Converterbased Wind Generation and Grids. IEEE J. Emerg. Sel. Top. Power Electron. 2020. [CrossRef]

20. Luo, J.; Bu, S.; Teng, F. An Optimal Modal Coordination Strategy based on Modal Superposition Theory to Mitigate Low Frequency Oscillation in FCWG Penetrated Power Systems. Int. J. Electr. Power Energy Syst. 2020, 120, 105975. [CrossRef]

21. Gautam, D.; Vittal, V.; Harbour, T. Impact of Increased Penetration of DFIG-Based Wind Turbine Generators on Transient and Small Signal Stability of Power Systems. IEEE Trans. Power Syst. 2009, 24, 1426-1434. [CrossRef]

22. Slootweg, J.G.; Kling, W.L. The impact of large scale wind power generation on power system oscillations. Electr. Power Syst. Res. 2003, 67, 9-20. [CrossRef]

23. Wen, B.; Boroyevich, D.; Burgos, R.; Mattavelli, P.; Shen, Z. Analysis of D-Q Small-Signal Impedance of Grid-Tied Inverters. IEEE Trans. Power Electron. 2016, 31, 675-687. [CrossRef]

24. Wang, L.; Lin, T.-C. Dynamic stability and transient responses of multiple grid-connected PV systems. In Proceedings of the 2008 IEEE/PES Transmission and Distribution Conference and Exposition, Chicago, IL, USA, 21-24 April 2008; pp. 1-6.

25. Teng, Y.; Zhang, X.; Fan, C.; Chen, X.; Zhang, R. Resonance Risk in Infirm-interconnected Grid Considering Photovoltaic Devices. In Proceedings of the 2018 International Conference on Power System Technology (POWERCON), Guangzhou, China, 6-8 November 2018; pp. 1004-1011.

26. Sun, J.; Li, M.; Zhang, Z.; Xu, T.; He, J.; Wang, H.; Li, G. Renewable Energy Transmission by HVDC Across the Continent: System Challenges and Opportunities. CSEE J. Power Energy Syst. 2017, 3, 353-364. [CrossRef]

27. Bi, J.T.; Du, W.; Wang, H.F. Aggregated dynamic model of grid-connected PV generation farms. In Proceedings of the International Conference on Renewable Power Generation (RPG 2015), Beijing, China, 7-18 October 2015.

28. Canizares, C.; Fernandes, T.; Geraldi, E.; Gerin-Lajoie, L.; Gibbard, M.; Hiskens, I.; Kersulis, J.; Kuiava, R.; Lima, L.; DeMarco, F.; et al. Benchmark Models for the Analysis and Control of Small-Signal Oscillatory Dynamics in Power Systems. IEEE Trans. Power Syst. 2017, 32, 715-722. [CrossRef] 